\title{
Testing the Assumptions Underlying Ocean Mixing Methodologies Using Direct Numerical Simulations
}

\author{
J. R. TAYLOR \\ Department of Applied Mathematics and Theoretical Physics, University of Cambridge, \\ Cambridge, United Kingdom \\ S. M. DE BRUYN KOPS \\ Department of Mechanical and Industrial Engineering, University of \\ Massachusetts Amherst, Amherst, Massachusetts \\ C. P. CAUlfield \\ BP Institute, and Department of Applied Mathematics and Theoretical Physics, University of \\ Cambridge, Cambridge, United Kingdom \\ P. F. LINDEN \\ Department of Applied Mathematics and Theoretical Physics, University of Cambridge, \\ Cambridge, United Kingdom
}

(Manuscript received 7 February 2019, in final form 22 July 2019)

\begin{abstract}
Direct numerical simulations of stratified turbulence are used to test several fundamental assumptions involved in the Osborn, Osborn-Cox, and Thorpe methods commonly used to estimate the turbulent diffusivity from field measurements. The forced simulations in an idealized triply periodic computational domain exhibit characteristic features of stratified turbulence including intermittency and layer formation. When calculated using the volume-averaged dissipation rates from the simulations, the vertical diffusivities inferred from the Osborn and Osborn-Cox methods are within $40 \%$ of the value diagnosed using the volume-averaged buoyancy flux for all cases, while the Thorpe-scale method performs similarly well in the simulation with a relatively large buoyancy Reynolds number $\left(\operatorname{Re}_{b} \simeq 240\right)$ but significantly overestimates the vertical diffusivity in simulations with $\mathrm{Re}_{b}<60$. The methods are also tested using a limited number of vertical profiles randomly selected from the computational volume. The Osborn, Osborn-Cox, and Thorpe-scale methods converge to their respective estimates based on volume-averaged statistics faster than the vertical diffusivity calculated directly from the buoyancy flux, which is contaminated with reversible contributions from internal waves. When applied to a small number of vertical profiles, several assumptions underlying the Osborn and OsbornCox methods are not well supported by the simulation data. However, the vertical diffusivity inferred from these methods compares reasonably well to the exact value from the simulations and outperforms the assumptions underlying these methods in terms of the relative error. Motivated by a recent theoretical development, it is speculated that the Osborn method might provide a reasonable approximation to the diffusivity associated with the irreversible buoyancy flux.
\end{abstract}

\section{Introduction}

Small-scale turbulence, defined here as three-dimensional overturning motions, plays an important role in setting the large-scale properties and circulation of the ocean.

Corresponding author: John R. Taylor, j.r.taylor@damtp. cam.ac.uk
Turbulence influences the depth of the surface and bottom mixed layers by entraining stratified water into the mixed layer (e.g., Large et al. 1994; Pacanowski and Philander 1981) thereby influencing biological productivity and the exchanges of heat and carbon between the atmosphere and ocean (Marra et al. 1990). On long time scales, turbulence gradually mixes distinct water masses in the ocean interior, thereby influencing the pathways 
of the global overturning circulation (Wunsch and Ferrari 2004; Marshall and Speer 2012).

Here we use the term "mixing" to refer to the irreversible homogenization of a scalar quantity. This stands in contrast to "stirring," which refers to the down-scale transfer of scalar variance and the generation of structures such as filaments by turbulent motions. Mixing relies on molecular diffusion of the scalar substance (e.g., heat or salt) that occurs at very small scales, while stirring is inevitably associated with larger scales. For a statistically homogeneous turbulent flow, mixing occurs at scales close to the Batchelor scale, $L_{B}=L_{K} / \sqrt{\mathrm{Pr}}$, where $L_{K}=\left(\nu^{3} / \varepsilon\right)^{1 / 4}$ is the Kolmogorov scale, $\operatorname{Pr}=\nu / \kappa_{m}$ is the Prandtl (or Schmidt) number, $\nu$ is the kinematic viscosity of the fluid, $\kappa_{m}$ is the molecular scalar diffusivity, and $\varepsilon$ is the dissipation rate of kinetic energy. For typical open ocean conditions where $\varepsilon \simeq 10^{-10}-10^{-6} \mathrm{~m}^{2} \mathrm{~s}^{-3}$, the corresponding Kolmogorov scale is $L_{K} \simeq 1 \mathrm{~mm}-1 \mathrm{~cm}$, and the thermal Batchelor scale is $L_{B} \simeq 0.3-3 \mathrm{~mm}$ while the haline Batchelor scale is an order of magnitude smaller. The very small scales involved make it difficult, if not impossible, currently to resolve scalar mixing in measurements or models.

Due to the difficulty associated with resolving the small scales involved in scalar mixing, observational methods generally involve calculating various proxies for mixing. A near-universal assumption in the ocean mixing literature is that an ensemble of turbulent motions can be modeled through a turbulent diffusivity, defined as the ensemble-averaged scalar flux (in a particular coordinate direction) divided by the ensembleaveraged gradient (in an independently chosen direction). Although the turbulent diffusivity is a second rank tensor, our focus here will be on the vertical component, which we define as

$$
\kappa \equiv \frac{-\left\langle w^{\prime} c^{\prime}\right\rangle}{\partial\langle c\rangle / \partial z}
$$

where $w$ is the vertical velocity, $c$ is a scalar quantity, angle brackets indicate an unspecified averaging operator assumed to be equivalent to ensemble averaging, and primes denote departures from this average. Note that in some contexts (e.g., at fronts or in isopycnal coordinate ocean models) the diapycnal diffusivity might be more appropriate than the vertical diffusivity. In the simulations that will be analyzed here, the large-scale buoyancy gradient is aligned with the vertical direction, and hence the vertical and diapycnal diffusivities are equivalent by construction.

Indeed, estimating $\kappa$ is one of the central aims of the ocean mixing community. Perhaps the most direct approach is to measure the vertical turbulent scalar flux $\left\langle w^{\prime} c^{\prime}\right\rangle$ through simultaneous measurements of the vertical velocity and scalar concentration. While this method is in principle possible (e.g., Moum 1996), it can be extremely difficult to measure the vertical velocity accurately, and the correlation between the velocity and scalar concentration introduces another possible source of error. In addition, as we will see later, internal waves can induce a significant reversible contribution to the turbulent scalar flux and removing these contributions can be very difficult.

Other indirect methods of measuring the turbulent diffusivity necessarily rely on assumptions about the nature of small-scale turbulence. Indirect methods can be arranged in two categories: "finescale" methods and "microstructure" methods, each based around different assumptions. Several finescale methods rely on the assumption that small-scale turbulence in the ocean interior is forced by the ambient internal wave field. These methods then link the mixing via small-scale turbulence with the properties of the internal wave field (e.g., Henyey et al. 1986; Gregg 1989; Polzin et al. 1995; MacKinnon and Gregg 2003).

Rather than relying on measurements of internal waves, microstructure methods use measurements of small-scale turbulence to infer the turbulent diffusivity. Two prominent microstructure methods are the OsbornCox method (Osborn and Cox 1972), which uses measurements of temperature or salinity variance and infers the scalar variance dissipation rate and diffusivity, and the Osborn method (Osborn 1980), which relates measurements of shear to the turbulent dissipation rate, and hence to the diffusivity. Gregg et al. (2018) provide a review and discussion of microstructure methods and their underlying assumptions.

An additional method for inferring the rate of mixing is the Thorpe-scale method. This method is perhaps best classified as intermediate between finescale and microstruture methods as it uses measurements of the scalar fields to infer the size of the largest turbulent motions. In this method unstable "overturns" in a measured temperature, salinity, or density profile are first related to the dissipation rate and then to the turbulent diffusivity following the Osborn method (Osborn 1980). These methods and their underlying assumptions will be described in more detail in section $3 \mathrm{c}$ below.

The primary objective of this paper is to evaluate microstructure and Thorpe-scale methods using output from direct numerical simulations (DNS) of forced stratified turbulence. By definition a DNS resolves all scales of turbulent motion. The simulations here have a molecular Prandtl number $\operatorname{Pr}=7$, a typical value corresponding to the diffusion of heat in seawater. Hence, the resolution of the simulations must be sufficient to 
capture scales near the Batchelor scale $(\sim 1 \mathrm{~mm}$ in dimensional terms). Our aim is to simulate typical turbulent conditions in the ocean interior. Even with a limited domain size, this makes the simulations extremely computationally expensive-here the simulations exceed $10^{12}$ grid points. The advantage of DNS is that turbulent quantities such as the dissipation rate and scalar flux can be evaluated exactly. This allows us to distinguish between uncertainties associated with measurement techniques from uncertainties associated with the underlying assumptions inherent in each method. Here, our focus is on such assumption-associated uncertainties.

The DNS that are analyzed here simulate turbulence in a relatively small $(\sim 5-10 \mathrm{~m})$ three-dimensional domain. Periodic boundary conditions are applied to the velocity in all three directions, while a constant vertical background stratification is imposed. The computational domain can be interpreted as a small region embedded in the ocean interior. The simulations are forced by applying a scale-selective deterministic body force to the momentum equations to energize the large scales of the horizontal velocity. While the forcing term is intended to represent energy input from uncaptured large-scale motions, we do not attempt to simulate a particular internal wave spectrum at the large scales. We therefore do not attempt to test any finescale parameterizations and instead focus on microstructure and Thorpe-scale-based methods.

Many microstructure measurement techniques involve fitting a canonical spectrum to the measured spectrum obtained from a depth window (Gregg 1999) or spatially averaging over a prescribed depth interval (Moum et al. 1995) or an identified turbulent patch (Moum 1996). This effectively produces one value of dissipation or diffusivity for a given depth interval. Similarly, the Thorpe-scale method requires the calculation of the root-mean-square (rms) displacement scale with respect to a finite depth window. In section $3 \mathrm{~d}$ we will apply the Osborn, Osborn-Cox, and Thorpe-scale methods to quantities calculated from vertical profiles extracted from the DNS, which generically can cover more than one "patch" of turbulence in any single profile.

Turbulence in strongly stratified fluids is often highly intermittent in space and time (see, e.g., Rorai et al. 2014; Portwood et al. 2016). This raises the following question: how well can a limited set of observations reproduce the volumetrically averaged turbulent diffusivity? In section $3 \mathrm{~d}$, we will also address this question by calculating the turbulent diffusivity with a limited number of vertical profiles extracted from the DNS. This can be interpreted as a best case scenario for observations of turbulent mixing without any measurement errors. In section 4, we discuss our results and draw some conclusions.

\section{Simulation setup and methodology}

\section{a. Governing equations}

The objective of the DNS is to simulate stratified turbulence in a quasi-equilibrated state where the energy input from large-scale forcing is balanced by small-scale dissipation and mixing. Periodic boundary conditions are applied in all three spatial directions, the details of which are given below. We do not directly consider the influence of any physical boundary and hence the computational domain can be viewed as a relatively small box embedded within the water column.

The simulations solve the nonhydrostatic Boussinesq equations that can be written in nondimensional form normalized by a characteristic velocity scale $U$, length scale $L$, and background buoyancy frequency $N_{0}$. The nondimensional equations are

$$
\begin{aligned}
\nabla \cdot \mathbf{u}= & 0, \\
\frac{\partial \mathbf{u}}{\partial t}+\mathbf{u} \cdot \nabla \mathbf{u}= & -\left(\frac{1}{\mathrm{Fr}}\right)^{2} \rho \hat{\mathbf{z}}-\nabla p \\
& +\frac{1}{\operatorname{Re}} \nabla^{2} \mathbf{u}+\mathscr{F}, \quad \text { and } \\
\frac{\partial b}{\partial t}+\mathbf{u} \cdot \nabla b+w= & \frac{1}{\operatorname{RePr}} \nabla^{2} b,
\end{aligned}
$$

where the nondimensional parameters are a characteristic Froude number, the Prandtl number, and a characteristic Reynolds number, defined respectively as

$$
\operatorname{Fr} \equiv \frac{U}{N_{0} L}, \quad \operatorname{Pr} \equiv \frac{\nu}{\kappa_{m}}, \quad \text { and } \quad \operatorname{Re} \equiv \frac{U L}{\nu} .
$$

Note that the diffusion of the scalar is specified by a characteristic Péclet number Pe $\equiv U L / \kappa_{m}=\operatorname{RePr}$. The buoyancy, $b \equiv-g \rho / \rho_{0}$ can be related to temperature through a linear equation of state, $b=\alpha g\left(T-T_{0}\right)$, where $\rho_{0}$ and $T_{0}$ are a reference density and temperature, respectively, and $\alpha$ is the thermal expansion coefficient. The buoyancy $b$ in Eq. (2c) is defined as the departure from an imposed background gradient such that the total buoyancy is $b_{T}=b+N_{0}^{2} z$. Periodic boundary conditions are then applied to $b$. In effect, this maintains a constant buoyancy difference between the top and bottom of the computational domain.

The periodic boundary conditions that are used here have implications for the flow that can develop. First, the relatively small domain size limits the scale of the motions that we are able to simulate directly. The body 
TABLE 1. Nondimensional simulation parameters and derived quantities.

\begin{tabular}{lccrccccccrrr}
\hline \hline Label & $\tilde{L}_{x, y}$ & $\tilde{L}_{z}$ & \multicolumn{1}{c}{$N_{x, y}$} & $N_{z}$ & $\mathrm{Re}$ & $\mathrm{Fr}$ & $\operatorname{Pr}$ & $\mathrm{Fr}_{h}$ & $\mathrm{Fr}_{t}$ & \multicolumn{1}{c}{$\operatorname{Re}_{h}$} & $\operatorname{Re}_{t}$ & $\operatorname{Re}_{b}$ \\
\hline $\mathrm{A}$ & $2 \pi$ & $\pi / 4$ & 9216 & 1152 & 6452 & 0.0416 & 7 & 0.071 & 0.0019 & 7048 & 82755 & 12.1 \\
$\mathrm{~B}$ & $2 \pi$ & $\pi / 4$ & 18432 & 2304 & 2410 & 0.0416 & 7 & 0.080 & 0.0025 & 23069 & 231575 & 57.5 \\
$\mathrm{C}$ & $2 \pi$ & $\pi$ & 13104 & 6552 & 4679 & 0.1667 & 7 & 0.45 & 0.015 & 2985 & 25597 & 241.5 \\
\hline
\end{tabular}

force [ $\mathscr{F}$ in Eq. (2b)] is meant to mimic the downscale transfer of momentum and energy from motions that are larger than our computational domain, albeit in an idealized way. The periodic boundary conditions applied to the velocity and the departures from the background stratification imply that the local momentum and buoyancy fluxes at the top of the computational domain match the values at the bottom of the computational domain. However, these fluxes do not need to remain constant within the domain. As a result (and as we will see below), the simulations develop layers with relatively weak and strong stratification and the vertical shear associated with the horizontally averaged velocity is nonzero.

\section{b. Numerical methods}

Equations (2) are solved in a triply periodic domain with the pseudospectral technique discussed in Almalkie and de Bruyn Kops (2012b). Spatial derivatives are computed in Fourier space, the nonlinear terms are computed in real space, and the solution is advanced in time in Fourier space with the variable-step, third-order, Adams-Bashforth algorithm with pressure projection. The nonlinear term in the momentum equation is computed in rotational form, and the advective term in the internal energy equation is computed in conservation and advective forms on alternate time steps. These techniques are standard to ensure conservation of energy and to eliminate most aliasing errors, but the simulations reported in this paper are fully dealiased in accordance with the $2 / 3$ rule via a spectral cutoff filter.

The body force $\mathscr{F}$ in Eq. (2) is implemented using the deterministic forcing schema denoted $\mathrm{Rf}$ in Rao and de Bruyn Kops (2011). The objective is to force all the simulations to have the same spectra $E_{h}\left(\kappa_{h}, \kappa_{z}\right)$ with $\kappa_{h}<\kappa_{z}$ and $\kappa_{z}=0$. The term $E_{h}$ is the power spectrum of the horizontal contribution to kinetic energy averaged over annuli of constant horizontal wavenumber $\kappa_{h}$ and vertical wavenumber $\kappa_{z}$. The highest wavenumber forced is $\kappa_{f}=16 \pi / L_{h}$, with $L_{h}$ the horizontal dimension of the numerical domain. Deterministic forcing requires choosing a target spectrum $E_{f}\left(\kappa_{h}<\kappa_{f}, 0\right)$. In contrast to turbulence that is isotropic and homogeneous in three dimensions, there are no theoretical model spectra for $E_{f}$ (cf. Overholt and Pope 1998). Therefore, run 2 from Lindborg (2006) was rerun using a stochastic forcing schema similar to that used by Lindborg and denoted schema Qg in Rao and de Bruyn Kops (2011). The spectrum for $E_{h}\left(\kappa_{h}<\kappa_{f}, 0\right)$ was computed from this simulation and used as the target for the simulations reported in the current paper.

In addition to forcing the large horizontal scales, $1 \%$ of the forcing energy is applied stochastically to the horizontal velocity components through wavenumber modes with $\kappa_{h}=0$ and $\kappa_{z}=2 \pi j / L_{v}, j=2,3,4$. Here $L_{v}$ is the vertical dimension of the numerical domain. This random forcing induces some vertical shear (Lindborg 2006). There is no forcing of the vertical velocity in the simulations.

The extents of the domain in the horizontal and vertical directions are $L_{h}$ and $L_{v}$ with $L_{h} / L_{v}$ chosen to accommodate the vertical motions that develop in the flow. While the simulation domains are not cubes and the vertical extent of the domain varies with the chosen characteristic Froude number, the grid spacing $\Delta$ is the same in all directions. It is assumed for the purpose of choosing the resolution of the numerical grid that the flows are approximately isotropic at the smallest length scales in the simulation. Therefore, a three-dimensional grid with spacing $\Delta=L_{h} / N_{x}=L_{h} / N_{y}=L_{v} / N_{z}$ with $N_{x}$, $N_{y}$, and $N_{z}$ being the number of grid points in the $x, y$, and $z$ directions, respectively, is used and any smallscale anisotropy in the flows can be attributed to flow physics rather than to numerical artifacts of an anisotropic grid (cf. Waite 2011).

\section{c. Parameters}

Three simulations (labeled A, B and C) are analyzed here, and the related nondimensional parameters are listed in Table 1. In each case the nondimensional horizontal domain size is $2 \pi$. Simulations A and B have the same characteristic Froude number, Fr $=0.0416$, representing relatively strong stratification. The Reynolds number is larger in simulation A compared to simulation B. Simulation $\mathrm{C}$ has a moderate Reynolds number and a larger characteristic Froude number representing weaker stratification.

Equations (2) are time-stepped until a statistically steady state is reached. The simulations can be described using nondimensional parameters derived using turbulent properties in the final state. For this purpose it is useful to define the turbulent kinetic energy (TKE) 
TABLE 2. Dimensional simulation parameters and derived quantities. The values here have been made dimensional by setting the vertical domain height $L_{z}=5 \mathrm{~m}$ and kinematic viscosity $\nu=10^{-6} \mathrm{~m}^{2} \mathrm{~s}^{-1}$ in each simulation.

\begin{tabular}{|c|c|c|c|c|c|c|c|c|}
\hline Label & $L_{x, y}$ & $L_{z}$ & $\Delta_{x, y, z}$ & $N_{0}^{2}$ & $\langle\varepsilon\rangle_{V}$ & $L_{O}^{V}$ & $L_{K}^{V}$ & $\kappa_{d}^{V}$ \\
\hline A & $40 \mathrm{~m}$ & $5 \mathrm{~m}$ & $4.3 \mathrm{~mm}$ & $1.41 \times 10^{-5} \mathrm{~s}^{-2}$ & $1.71 \times 10^{-10} \mathrm{~m}^{2} \mathrm{~s}^{-3}$ & $5.6 \mathrm{~cm}$ & $8.7 \mathrm{~mm}$ & $2.2 \times 10^{-6} \mathrm{~m}^{2} \mathrm{~s}^{-1}$ \\
\hline B & $40 \mathrm{~m}$ & $5 \mathrm{~m}$ & $2.2 \mathrm{~mm}$ & $2.00 \times 10^{-4} \mathrm{~s}^{-2}$ & $1.15 \times 10^{-8} \mathrm{~m}^{2} \mathrm{~s}^{-3}$ & $6.3 \mathrm{~cm}$ & $3.0 \mathrm{~mm}$ & $1.8 \times 10^{-5} \mathrm{~m}^{2} \mathrm{~s}^{-1}$ \\
\hline $\mathrm{C}$ & $10 \mathrm{~m}$ & $5 \mathrm{~m}$ & $0.76 \mathrm{~mm}$ & $1.23 \times 10^{-4} \mathrm{~s}^{-2}$ & $2.97 \times 10^{-8} \mathrm{~m}^{2} \mathrm{~s}^{-3}$ & $14.8 \mathrm{~cm}$ & $2.4 \mathrm{~mm}$ & $7.2 \times 10^{-5} \mathrm{~m}^{2} \mathrm{~s}^{-1}$ \\
\hline
\end{tabular}

$k \equiv\left\langle\mathbf{u}^{\prime} \cdot \mathbf{u}^{\prime}\right\rangle_{V}^{1 / 2} / 2$ and the TKE dissipation rate $\langle\varepsilon\rangle_{V} \equiv$ $2 \nu\left\langle s_{i j} s_{i j}\right\rangle_{V}$, where

$$
s_{i j} \equiv \frac{1}{2}\left(\frac{\partial u_{i}^{\prime}}{\partial x_{j}}+\frac{\partial u_{j}^{\prime}}{\partial x_{i}}\right)
$$

is the fluctuating rate of strain tensor, $\langle\cdot\rangle_{V}$ denotes an average over the full computational volume, and primes denote departures from this volume average. The Reynolds number of the turbulent flow can then be characterized using the horizontal $\mathrm{rms}$ velocity $u_{\mathrm{rms}} \equiv\left\langle\mathbf{u}_{h}^{\prime} \cdot \mathbf{u}_{h}^{\prime}\right\rangle_{V}^{1 / 2}$ and a characteristic length scale. Two choices for the length scale are the integral length scale $L_{i}$ and the turbulent length scale $L_{t} \equiv\langle k\rangle_{V}^{3 / 2} /\langle\varepsilon\rangle_{V}$, thereby forming two derived Reynolds numbers,

$$
\operatorname{Re}_{h} \equiv \frac{u_{\mathrm{rms}} L_{i}}{\nu}, \quad \text { and } \quad \operatorname{Re}_{t} \equiv \frac{u_{\mathrm{rms}} L_{t}}{\nu}
$$

Here $L_{i}$ is computed from the longitudinal horizontal velocity spectra using the method of Comte-Bellot and Corrsin (1971, see their appendix E). Similarly, the relative strength of stratification can be quantified by two derived Froude numbers,

$$
\operatorname{Fr}_{h} \equiv \frac{u_{\mathrm{rms}}}{N_{0} L_{i}}, \quad \text { and } \quad \mathrm{Fr}_{t} \equiv \frac{u_{\mathrm{rms}}}{N_{0} L_{t}}
$$

The integral scale $L_{i}$ is a direct estimate of the length scale of the motions responsible for most of the kinetic energy in a flow. Since calculation of $L_{i}$ requires two point statistics to compute, $L_{t}$ has long been used as a surrogate, and we provide it here to facilitate comparisons with other data. For isotropic homogeneous turbulence, $\mathscr{D} \equiv L_{i} / L_{t} \approx 0.5$ (Pope 2000), and for decaying unstratified turbulence it has been observed to be as high as 1.81 (Sreenivasan 1998; Wang et al. 1996). For stratified turbulence with unity $\operatorname{Pr}, \mathscr{D}$ ranges from 0.3 to 0.5 (de Bruyn Kops 2015; Maffioli and Davidson 2016) and decreases with decreasing buoyancy Reynolds number (defined in the next paragraph) (de Bruyn Kops and Riley 2019). In the current simulations with $\operatorname{Pr}=7, \mathscr{D}$ is approximately 0.1 .

Stratification and viscosity can both act to inhibit turbulence motions. The combination of these effects can be quantified using a buoyancy Reynolds number (also referred to as a turbulent activity coefficient; Dillon and Caldwell 1980; Gibson 1980),

$$
\operatorname{Re}_{b} \equiv \frac{\langle\varepsilon\rangle_{V}}{\nu N_{0}^{2}} .
$$

From this definition, the buoyancy Reynolds number can be related to a ratio of Ozmidov and Kolmogorov scales, $\operatorname{Re}_{b}=\left(L_{O}^{V} / L_{K}^{V}\right)^{4 / 3}$, where

$$
L_{K}^{V} \equiv\left(\frac{\nu^{3}}{\langle\varepsilon\rangle_{V}}\right)^{1 / 4}, \quad \text { and } \quad L_{O}^{V} \equiv \frac{\langle\varepsilon\rangle_{V}^{1 / 2}}{N_{0}^{3 / 2}} .
$$

Loosely, the Ozmidov scale characterizes the size of the largest turbulent overturns permitted by stratification and the Kolmogorov scale characterizes the size of the smallest motions permitted by viscosity. Therefore, $\mathrm{Re}_{b}$ provides a measure of the dynamic range associated with turbulent overturning motions, largely unaffected by either buoyancy or viscosity. The simulations in Table 1 are listed in order of increasing $\mathrm{Re}_{b}$. Values of $\mathrm{Re}_{b}$ in this range (20-250) are common in the ocean interior according to a recent estimate based on Argo data (Salehipour et al. 2016) and finescale parameterizations (Gregg 1989). Larger values of $\mathrm{Re}_{b}$ are also observed (Moum 1996), but these are not currently accessible with DNS of strongly stratified flows with realistic Pr.

For comparison with observations it is useful to construct a set of dimensional parameters for each simulation. Here, this is done by setting the dimensional vertical domain size to $5 \mathrm{~m}$ and the kinematic viscosity to $10^{-6} \mathrm{~m}^{2} \mathrm{~s}^{-1}$, appropriate for water. The dimensional domain size was chosen to match roughly the size of typical turbulent patches in the ocean interior and the vertical size typically used for averaging microstructure measurements (Moum 1996; Smyth et al. 2001). The horizontal dimensional domain size is $40 \mathrm{~m}$ in simulations $\mathrm{A}$ and $\mathrm{B}$ and $10 \mathrm{~m}$ in simulation C. As we will see, the domain size is sufficient to accommodate many turbulent overturns. For comparison the largest dimensional domain size used in the simulations of Smyth et al. (2001) (for $\operatorname{Pr}=7$ ) was $2.73 \mathrm{~m} \times 1.36 \mathrm{~m} \times 0.34 \mathrm{~m}$.

Once the dimensional domain size and kinematic viscosity are set, the dimensional time scale can be found from the characteristic Reynolds number Re. Some of the dimensional parameters are listed in Table 2. The dimensional values of the background buoyancy frequency, 
$N_{0}$, are in the range from $3.7 \times 10^{-3}$ to $1.4 \times 10^{-2} \mathrm{~s}^{-1}$, corresponding to buoyancy periods ranging from 28.0 to $7.4 \mathrm{~min}$. The weakest stratification considered here is within the range observed by Moum (1996) in the main thermocline while the strongest stratification considered here is more typical of the seasonal pycnocline (e.g., Alford and Pinkel 2000b). The dimensional average turbulent dissipation rate spans more than two orders of magnitude and contains values typically measured in the ocean interior (e.g., Moum 1996; Gregg 1989). The vertical turbulent diffusivity calculated with the volume-averaged vertical buoyancy flux $B \equiv w^{\prime} b^{\prime}$ is $\kappa_{d}^{V} \equiv-\langle B\rangle_{V} /\left\langle N^{2}\right\rangle_{V}$ ranges from $2.2 \times 10^{-6} \mathrm{~m}^{2} \mathrm{~s}^{-1}$ in simulation $A$ to $7.2 \times$ $10^{-5} \mathrm{~m}^{2} \mathrm{~s}^{-1}$ in simulation C. The small (close to molecular) diffusivity in simulation $\mathrm{A}$ is consistent with the observation by Ivey and Imberger (1991) that turbulence collapses for $\mathrm{Re}_{b} \lesssim 15$. However, as discussed by Rorai et al. (2014) and Portwood et al. (2016), strongly stratified turbulence is highly intermittent in space and time and (as we will see below) the volume-averaged statistics are not indicative of the turbulence at single points in space.

\section{Results}

\section{a. Vertical section and profiles}

Turbulence and mixing are intermittent across a wide range of scales in the DNS. On small scales, the statistics of energy and buoyancy variance dissipation are skewed with a small number of large events dominating the volume average. This is a well-known property of high Reynolds number turbulence in unstratified flows (Sreenivasan and Antonia 1997) and intermittency in scalar mixing is discussed extensively in Warhaft (2000). On larger scales, turbulence occurs in localized bursts separated by relatively quiescent flow. Similar behavior has been observed in numerous previous studies (e.g., Riley and de Bruyn Kops 2003; Hebert and de Bruyn Kops 2006a; Rorai et al. 2014; Portwood et al. 2016).

The top row in Fig. 1 shows a vertical cross section of buoyancy $b$ and the TKE dissipation rate $\varepsilon$ from simulation $\mathrm{C}$. The other simulations (not shown) have qualitatively similar features. A series of distinct layers are visible in the buoyancy field with relatively thick weakly stratified regions separated by relatively thin and more strongly stratified interfaces. The turbulent dissipation rate exhibits localized patches of strong turbulence similar to those described in Portwood et al. (2016). Maximum local values of $\varepsilon$ are up to 30 times larger than the volume average.

The lower panels in Fig. 1 show a close-up view of the flow in the boxed regions labeled 1,2, and 3 in the top panels. To quantify mixing in each region, it is convenient to introduce the perturbation potential energy. In a volume with constant background buoyancy gradient $N_{0}^{2}$, the perturbation potential energy is $\left\langle b^{\prime 2}\right\rangle_{V} /\left(2 N_{0}^{2}\right)$ and its associated dissipation rate can be written as

$$
\chi \equiv \frac{\kappa_{m} \nabla b^{\prime} \cdot \nabla b^{\prime}}{N_{0}^{2}} .
$$

Since $N_{0}^{2}$ is constant in our simulations, $\chi$ is proportional to the dissipation rate of buoyancy variance, and hence is a natural measure of irreversible mixing [see Salehipour and Peltier (2015) for a detailed discussion].

Region 1 is associated with relatively large kinetic and potential energy dissipation rates. As seen in the buoyancy field, in the middle of this region is a $\sim 0.5-\mathrm{m}$ vertical overturn. At the center of the overturn $\chi$ is relatively weak while $\varepsilon$ remains large. Along the edges of the overturn at this instant in time $\chi$ and $\varepsilon$ are of similar magnitude. In other words, mixing is more efficient on the flanks of the overturn than in the center of the overturn.

Region 2 exhibits a moderate value of $\varepsilon$ and an undulating density interface passes through the region. While $\varepsilon$ is relatively uniform in the region, $\chi$ is significantly larger near the density interface than in the mixed regions above and below the interface. Small overturns, $5-10 \mathrm{~cm}$ in height, appear along the density interface, but these features appear irregular.

Region 3 is characterized by relatively small values of $\varepsilon$ and a relatively flat density interface. A vertically sheared flow exists on either side of the density interface and a series of what appear to be shear-induced billows can be seen. These billow-like structures are highlighted by relatively large values of $\chi$.

Statistics collected along a single vertical profile corresponding to the white dashed line in Fig. 1 are shown in Fig. 2. The red dashed line in Fig. 2a shows the 1D buoyancy profile sorted so that buoyancy increases monotonically with height. The displacement scale $L_{d}$ is the change in height of a fluid parcel from its unsorted to sorted positions. Several features in the profiles shown in Fig. 2 resemble qualitatively the observed profiles reported in Moum (1996) such as the step-like structure in the density field and the corresponding structure in the Thorpe displacement scale (see, e.g., Fig. 1b in Moum 1996). The buoyancy flux $w^{\prime} b^{\prime}$ alternates in sign along the vertical profile, indicating reversible transfer between perturbation potential and kinetic energy.

The kinetic and potential energy dissipation rates are highly intermittent (see Figs. $2 \mathrm{~d}$ and $2 \mathrm{e}$ ). There is no clear correlation between locations with large $\varepsilon$ and $\chi$. 

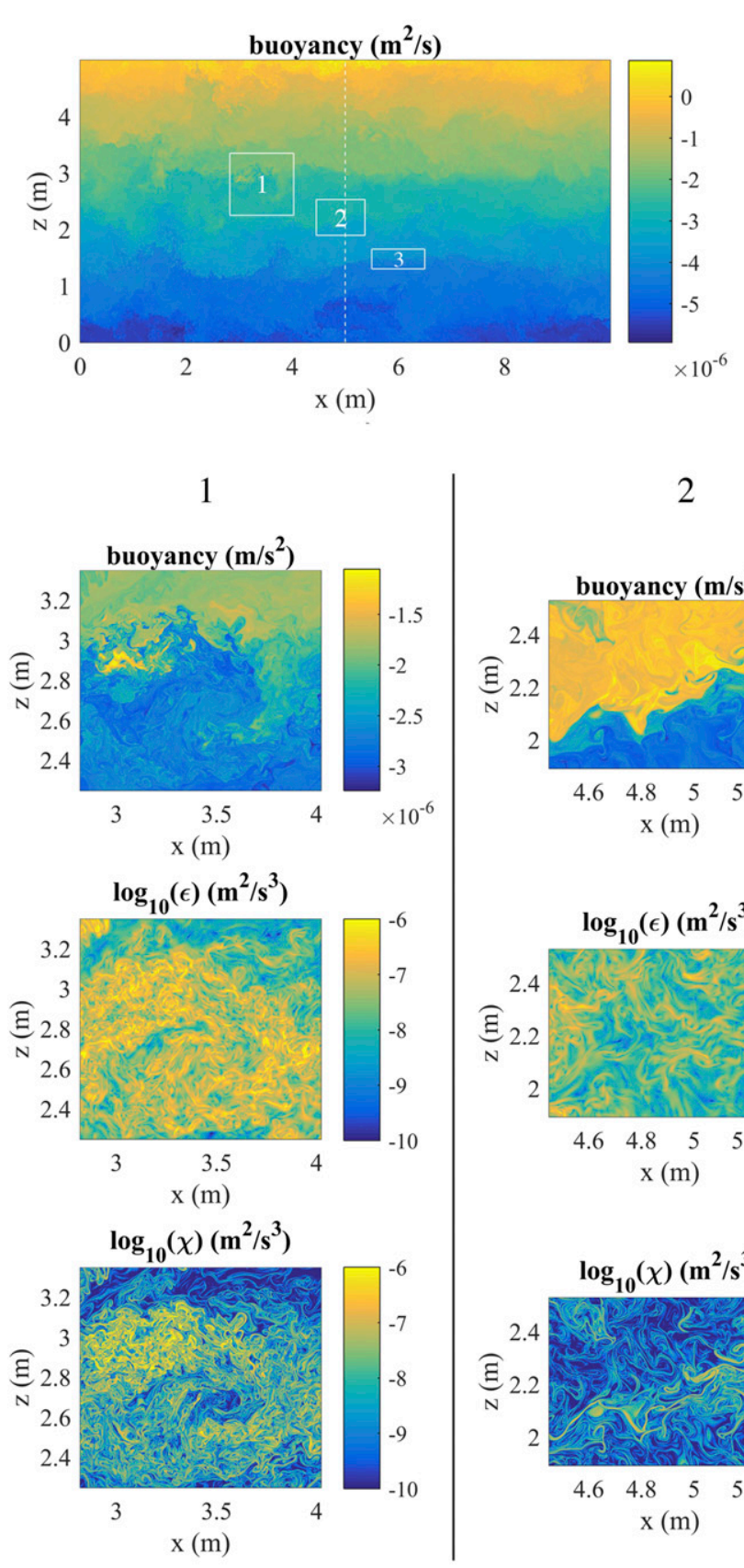

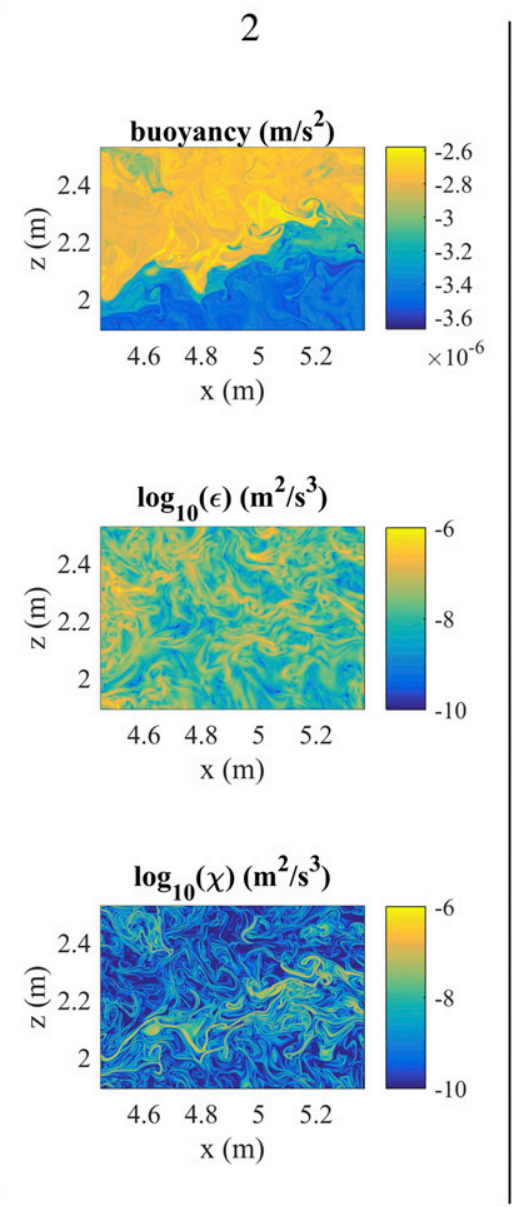

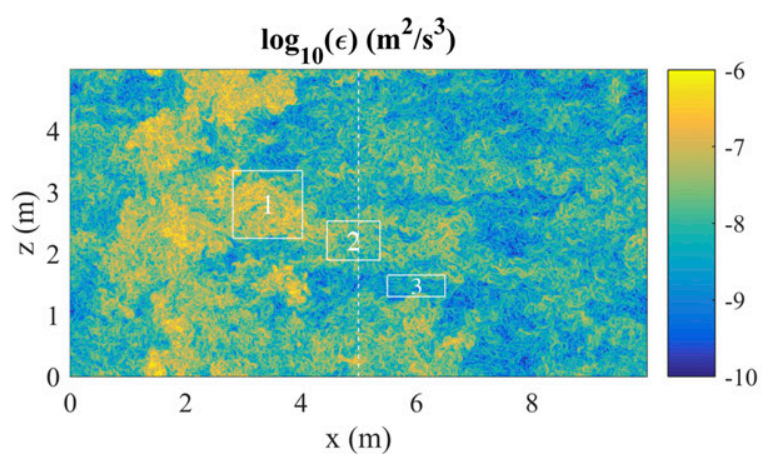

3
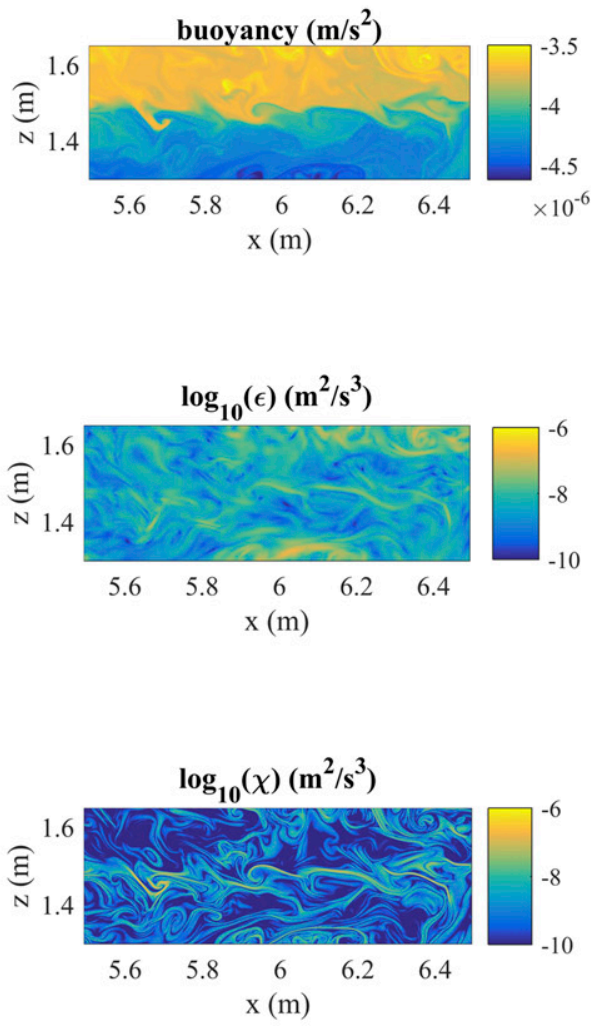

FIG. 1. (top) Dimensional buoyancy and turbulent kinetic energy dissipation rate $\varepsilon$ on a vertical 2D slice extracted from simulation C. Panels in columns 1, 2, and 3 show a close-up of regions with strong, moderate, and weak dissipation as indicated by the boxed regions labeled in the top row. The dissipation rate of perturbation potential energy $\chi$ is also shown. The white dashed lines in the top row indicate the location of the profile shown in Fig. 2.

As a result, a local mixing efficiency $\eta(\mathbf{x}, t)$, which may be defined as

$$
\eta(\mathbf{x}, t) \equiv \frac{\chi}{\chi+\varepsilon},
$$

fluctuates rapidly between 0 and 1 (Fig. 2f).

\section{b. Length scales}

The relative importance of stratification and viscosity to the turbulent motions at a particular scale can be quantified by comparing various length scales associated with stratified turbulence (Smyth and Moum 2000). Figure 3 shows characteristic length scales for each 
(a)

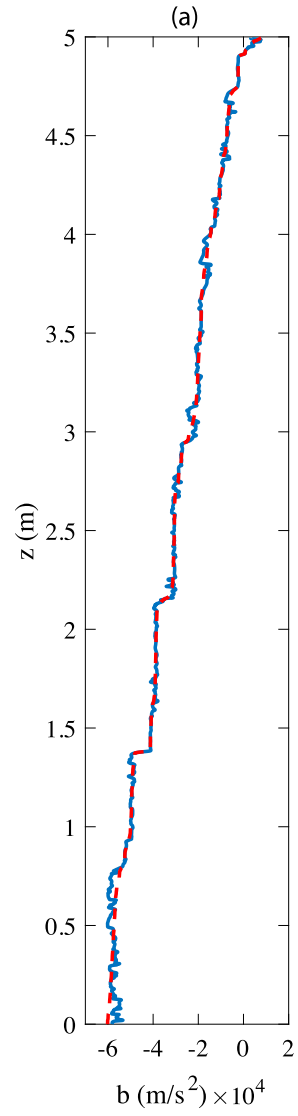

(b)

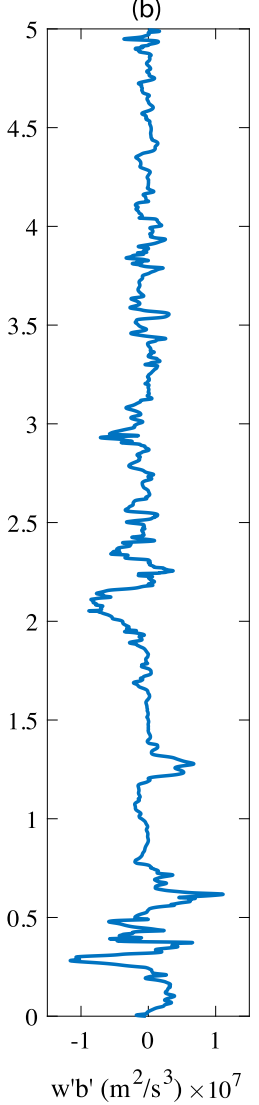

(c)

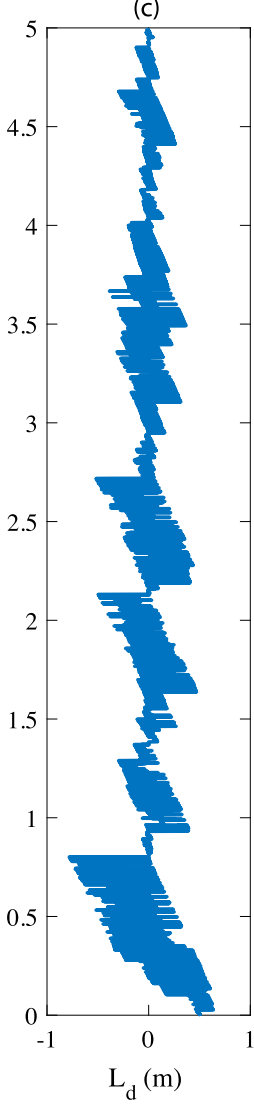

(d)

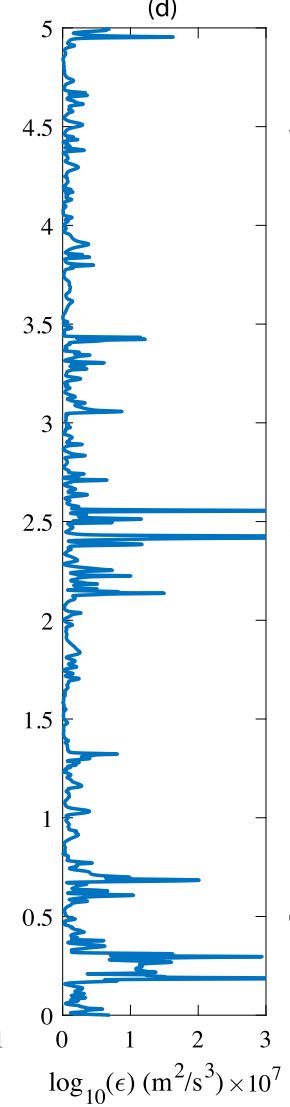

(e)

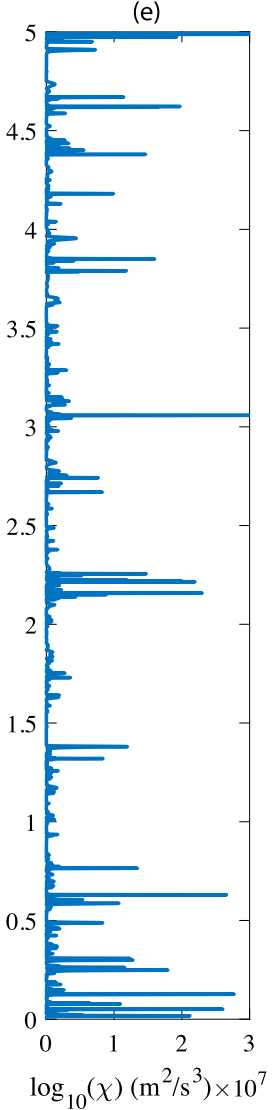

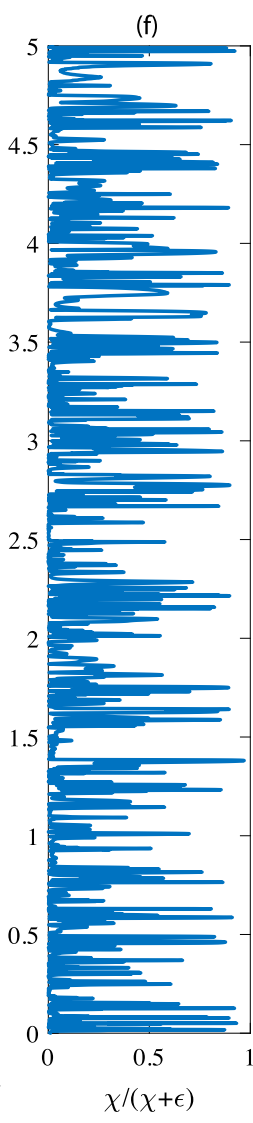

FIG. 2. Vertical profiles of (a) buoyancy, (b) buoyancy flux, (c) displacement scale $L_{d}$, (d) turbulent kinetic energy dissipation rate, (e) turbulent potential energy dissipation rate, and (f) local mixing efficiency extracted from simulation C. The profiles are taken from the location indicated with a white dashed line in Fig. 1. The dashed red line in (a) is the sorted density profile.

simulation, plotted as a function of the buoyancy Reynolds number, $\mathrm{Re}_{b}$. Here, dimensional values are plotted, where the vertical domain size is set to $5 \mathrm{~m}$ as discussed above.

In dimensional terms, the Kolmogorov scale, $L_{K}$, ranges from 2.4 to $8.7 \mathrm{~mm}$, while the Batchelor scale, $L_{B}=L_{K} / \sqrt{\operatorname{Pr}}$, ranges from 0.9 to $3.3 \mathrm{~mm}$. The isotropic grid spacing $\Delta_{x, y, z}$ is always less than twice the Batchelor scale, ensuring that the DNS is sufficiently well resolved. The wide scale separation between the domain size and the grid spacing gives an indication of the large computational cost of these simulations.

There are several different ways to construct a Thorpe scale from a three-dimensional dataset [see Smyth and Moum (2000) and Mashayek et al. (2017a) for further discussion]. For example, it would be possible to sort a three-dimensional density field (e.g., Winters and D'Asaro 1996) and calculate the Thorpe scale from the rms vertical displacements with respect to the volumetrically sorted profile. Here, motivated by oceanographic observations where three-dimensional sorting is typically not possible, we instead vertically sort the density profile at each horizontal grid point. The Thorpe scale is then calculated from each vertical profile and the result shown in Fig. 3 is averaged over all horizontal grid points. Specifically,

$$
L_{T}^{V} \equiv\left\langle\left\langle L_{d}^{2}\right\rangle_{z}^{1 / 2}\right\rangle_{x, y}
$$

where $\langle\cdot\rangle_{z}$ denotes an average in the vertical direction and $\langle\cdot\rangle_{x, y}$ denotes an average in the horizontal directions. Later, in section 3d, we will examine the sensitivity of the Thorpe-scale estimates calculated with a limited number of vertical profiles.

The dimensional Thorpe and Ozmidov scales calculated using volumetric simulation data, $L_{T}^{V}$ and $L_{O}^{V}$ are both $\simeq 10 \mathrm{~cm}$ and increase somewhat with increasing buoyancy Reynolds number. The Ozmidov scale increases with $\mathrm{Re}_{b}$ faster than the Thorpe scale such that the ratio $L_{O}^{V} / L_{T}^{V}$ is 0.53 in simulation A, 0.56 in simulation $\mathrm{B}$, and 0.92 in simulation C. This can be compared with $L_{O} / L_{T} \simeq 0.8$ suggested by Dillon and Caldwell (1980). The dependence of this ratio on the flow parameters is consistent with the recent conclusions of Mater et al. (2015) and Scotti (2015). 


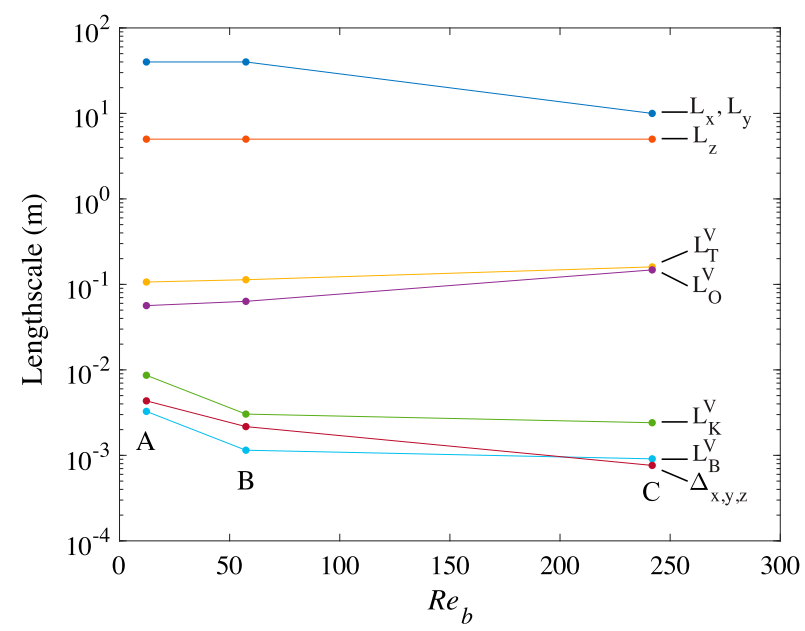

FIG. 3. Dimensional length scales: horizontal domain size $L_{x}, L_{y}$, vertical domain size $L_{z}$, Thorpe scale $L_{T}^{V}$, Ozmidov scale $L_{O}^{V}$, Kolmogorov scale $L_{K}^{V}$, Batchelor scale $L_{B}^{V}$, and grid spacing $\Delta_{x, y, z}$. Simulation labels are given at the bottom of each series. The volume-averaged dissipation rate was used to calculate $L_{O}^{V}, L_{K}^{V}$, and $L_{B}^{V}$, and the Thorpe scale was calculated by sorting individual $1 \mathrm{D}$ density profiles and averaging the resulting Thorpe scale over all horizontal grid points.

\section{c. Testing of Osborn, Osborn-Cox, and Dillon methods}

In this section, we will compare the vertical turbulent diffusivity diagnosed directly from the simulations with values inferred from the Osborn, Osborn-Cox, and Dillon methods. Before giving the results, a brief description of each method is given below, highlighting in particular some of the key assumptions behind each method.

\section{1) OSBORN-COX METHOD}

Starting from an equation for entropy density, Osborn and Cox (1972) derived a method to estimate the vertical turbulent diffusivity from measurements of microscale temperature or conductivity. Here, we will write the equations in terms of buoyancy $b$ with the understanding that this is more closely related to temperature than salinity since the Prandtl number is 7 in the DNS. The buoyancy variance budget (as noted above this is linearly related to the perturbation potential energy in this context) can be written as

$$
\begin{gathered}
\left(\frac{\partial}{\partial t}+\langle\mathbf{u}\rangle \cdot \nabla\right)\left\langle b^{2}\right\rangle+\nabla \cdot\left(\left\langle\mathbf{u}^{\prime} b^{2}\right\rangle-\kappa_{m} \nabla\left\langle b^{\prime 2}\right\rangle\right) \\
\quad=-2\left\langle\mathbf{u}^{\prime} b^{\prime}\right\rangle \cdot \nabla\langle b\rangle-2 \kappa_{m}\left\langle\nabla b^{\prime} \cdot \nabla b^{\prime}\right\rangle
\end{gathered}
$$

where angle brackets denote an average over some arbitrary volume (e.g., Pope 2000). Assuming that terms on the left hand side, the time rate of change and flux divergence, are both small, Eq. (11) reduces to a productiondissipation balance

$$
-\left\langle\mathbf{u}^{\prime} b^{\prime}\right\rangle \cdot \nabla\langle b\rangle=\kappa_{m}\left\langle\nabla b^{\prime} \cdot \nabla b^{\prime}\right\rangle=\langle\chi\rangle\left\langle N^{2}\right\rangle,
$$

using Eq. (8). Further neglecting the horizontal buoyancy flux and defining the vertical diffusivity in terms of this (arbitrary) volume averages, that is, $\kappa \equiv-\langle B\rangle /\left\langle N^{2}\right\rangle$ yields an estimate of the vertical turbulent diffusivity,

$$
\kappa_{\mathrm{O}-\mathrm{C}} \equiv \frac{-\langle B\rangle}{\left\langle N^{2}\right\rangle} \simeq \frac{\langle\chi\rangle}{\left\langle N^{2}\right\rangle} .
$$

\section{2) OSBORN METHOD}

The Osborn method (Osborn 1980) provides a way to estimate the vertical diffusivity associated with smallscale turbulence from the TKE dissipation rate. In deriving the method, Osborn made several key assumptions [see, e.g., Mashayek et al. (2013) for further discussion], including that the vertical diffusivity is dominated by fully developed turbulence, and that the turbulence exhibits a quasi-steady balance between production, dissipation and diapycnal mixing when suitably averaged so that the mixing can be related to the dissipation rate. Therefore, the TKE budget reduces to a balance between production, buoyancy flux, and dissipation (with crucially no contribution from advective or boundary processes), that is,

$$
\langle P\rangle=\langle\varepsilon\rangle-\langle B\rangle,
$$

where

$$
\langle P\rangle \equiv-\left\langle u_{i}^{\prime} u_{j}^{\prime}\right\rangle \frac{\partial\left\langle u_{i}\right\rangle}{\partial x_{j}}
$$

is the turbulent shear production. Osborn (1980) further assumed that small-scale turbulence is isotropic so that the dissipation rate can be determined from just one component of the deformation rate tensor. We do not test this assumption here and instead evaluate the production and dissipation using the full deformation rate tensor. The appropriateness of the assumption of smallscale isotropy for stratified turbulence has been discussed extensively in recent papers (e.g., Hebert and de Bruyn Kops 2006b; Almalkie and de Bruyn Kops 2012a; de Bruyn Kops 2015). Osborn (1980) further suggested that the assumption of quasi-steadiness and hence the averaging operator could be applied to vertical profiles through turbulent patches ranging from 1 to $10 \mathrm{~m}$ in size.

Using the classical definition of the flux Richardson number, $R_{f} \equiv-\langle B\rangle /\langle P\rangle$ [or $R_{f}=\langle B\rangle /(\langle B\rangle-\langle\varepsilon\rangle)$ using 
Eq. (14)], the buoyancy flux may be expressed in terms of the TKE dissipation rate $\varepsilon$ as

$$
\langle B\rangle=-\left(\frac{R_{f}}{1-R_{f}}\right)\langle\varepsilon\rangle .
$$

Then, the vertical turbulent diffusivity, $\kappa=-\langle B\rangle /\left\langle N^{2}\right\rangle$, can be related to $\varepsilon$ to yield the estimate

$$
\kappa_{O}=\Gamma \frac{\langle\varepsilon\rangle}{\left\langle N^{2}\right\rangle},
$$

where $\Gamma \equiv\left[R_{f} /\left(1-R_{f}\right)\right]$. The turbulent flux coefficient $\Gamma$ is often referred to as a "mixing efficiency," although in principle it can be greater than one, and there has been much recent activity attempting to produce appropriate parameterizations for this quantity in terms of various flow parameters (see, e.g., Salehipour et al. 2016; Mashayek et al. 2017b; Monismith et al. 2018).

\section{3) THORPE-SCALE METHOD}

Thorpe (1977) proposed a method to estimate the averaged dissipation rate based on vertical profiles of potential density. An advantage of this method is that it can be applied to more readily available data (Gargett and Garner 2008). To calculate the Thorpe scale, a density profile is first sorted so that the sorted density is a monotonic decreasing function of height. The displacement length $L_{d}$ is the difference in height of a water parcel from its unsorted to sorted location (Fig. 2c). The Thorpe scale is then calculated by taking the rootmean-square of $L_{d}$, that is,

$$
L_{T}^{P}=\left\langle L_{d}^{2}\right\rangle_{P}^{1 / 2}
$$

where angle brackets are typically taken to represent an appropriate patch average, for example taken over a single overturning turbulent patch or an ensemble of such patches obtained from vertical profiling instruments (Thorpe 2005). Thorpe (1977) conjectured that $L_{T}^{P}$ may be linearly related to the Ozmidov scale calculated with patch-averaged quantities $L_{O}^{P}=\langle\varepsilon\rangle_{P}^{1 / 2}\left\langle N^{2}\right\rangle_{P}^{-3 / 2}$. This then gives an estimate of the dissipation rate

$$
\langle\varepsilon\rangle_{P}=R_{O T}^{2}\left(L_{T}^{P}\right)^{2}\left\langle N^{2}\right\rangle_{P}^{3 / 2},
$$

where the coefficient of proportionality $L_{O}^{P} / L_{T}^{P} \equiv R_{O T} \simeq 0.8$ is based on observations by Dillon and Caldwell (1980), although there is mounting evidence that estimates of this coefficient can be both biased and uncertain (Mater et al. 2015; Scotti 2015; Mashayek et al. 2017a). Then, using Eq. (17) yields an estimate for the vertical turbulent diffusivity,

$$
\kappa_{T}=0.64 \Gamma\left(L_{T}^{P}\right)^{2}\left\langle N^{2}\right\rangle_{P}^{1 / 2} .
$$

\section{4) COMPARISON}

The underlying assumptions behind the three methods described above are questionable in strongly stratified flows where turbulent events are highly intermittent in time and space as illustrated in Fig. 1. This concern becomes stronger when a small subset of the flow is sampled, for example using a small number of vertical profiles, since the various averages being taken become less reliable as representative of turbulent mixing events within the flow. Before addressing the issue of incomplete sampling and averaging, we will first examine the performance of the approximate methods described above, compared with the "direct" calculation of $\kappa$ formed using the volume-averaged buoyancy flux and stratification, that is,

$$
\kappa_{d}^{V}=\frac{-\langle B\rangle_{V}}{\left\langle N^{2}\right\rangle_{V}} .
$$

When calculated using data from the full computational volume, the vertical turbulent diffusivity associated with the Osborn-Cox, Osborn, and Thorpe methods can be written

$$
\begin{aligned}
\kappa_{\mathrm{O}-\mathrm{C}}^{V} & =\frac{\langle\chi\rangle_{V}}{\left\langle N^{2}\right\rangle_{V}}, \quad \kappa_{O}^{V}=\Gamma \frac{\langle\varepsilon\rangle_{V}}{\left\langle N^{2}\right\rangle_{V}}, \\
\kappa_{T}^{V} & =0.64 \Gamma\left(L_{T}^{V}\right)^{2}\left\langle N^{2}\right\rangle_{V}^{1 / 2}
\end{aligned}
$$

respectively, where $\langle\cdot\rangle_{V}$ denotes an average over the full computational volume and $L_{T}^{V}$ is defined in Eq. (10). Here we use $\Gamma=0.2$. Figure 4 shows $\kappa_{\mathrm{O}-\mathrm{C}}^{V}, \kappa_{O}^{V}$ and $\kappa_{T}^{V}$, normalized by $\kappa_{d}^{V}$ as defined in Eq. (21) and plotted against the buoyancy Reynolds number to differentiate the three simulations. The dimensional values of $\kappa_{d}^{V}$ are $2.2 \times 10^{-6} \mathrm{~m}^{2} \mathrm{~s}^{-1}$ in simulation A, $1.8 \times 10^{-5} \mathrm{~m}^{2} \mathrm{~s}^{-1}$ in simulation $\mathrm{B}$, and $7.2 \times 10^{-5} \mathrm{~m}^{2} \mathrm{~s}^{-1}$ in simulation $\mathrm{C}$, roughly spanning typical values found in the ocean interior (Waterhouse et al. 2014).

Even with perfect sampling of the 3D volume, there are significant differences between the various estimates of $\kappa$. The estimates using the Osborn and Osborn-Cox methods, $\kappa_{O}^{V}$ and $\kappa_{\mathrm{O} \text {-C }}^{V}$, are within $40 \%$ of $\kappa_{d}^{V}$, and there is no clear trend with $\mathrm{Re}_{b}$. The Thorpe-scale method underestimates $\kappa_{d}^{V}$ by about $50 \%$ in simulation $\mathrm{C}$, but significantly overestimates $\kappa_{d}^{V}$ in simulations A and B. Recall that our simulations are analyzed at a statistically steady state. It is possible that temporal variability could lead to larger biases when these methods are applied to oceanographic data. In addition, when the Thorpe scale is small and/or when the density contrast is weak, it can 


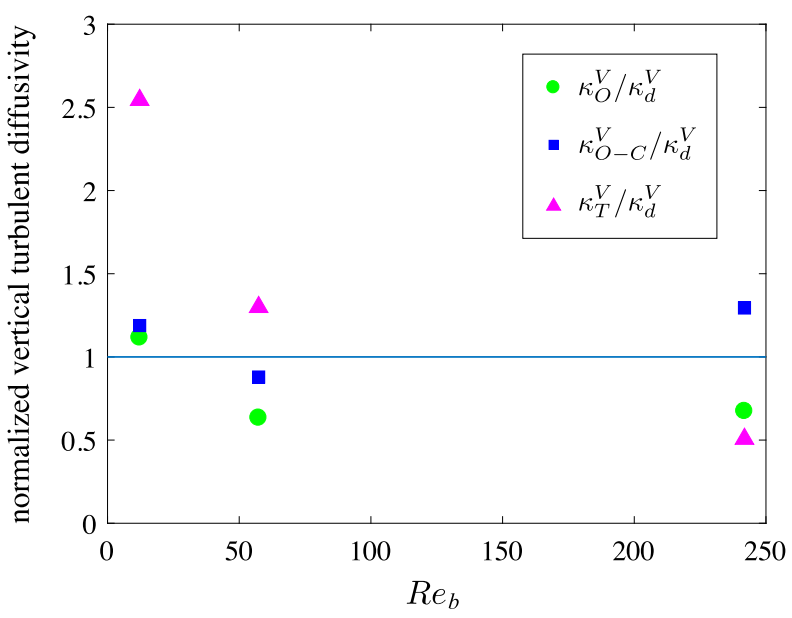

FIG. 4. Vertical diffusivity estimated using the Osborn method $\kappa_{O}^{V}$ (green circles), Osborn-Cox method $\kappa_{\text {O-C }}^{V}$ (blue squares), and Thorpe method $\kappa_{T}^{V}$ (magenta triangles), each calculated using data extracted from the full computational volume as defined in Eq. (22) and normalized by the turbulent vertical diffusivity diagnosed directly from the volume-averaged buoyancy flux $\kappa_{d}^{V}$.

be difficult to distinguish between real overturns and measurement error associated with a CTD profile (Ferron et al. 1998; Alford and Pinkel 2000a; Johnson and Garrett 2004).

\section{d. Vertical profile-averaged statistics}

The estimates of the vertical turbulent diffusivity described above were calculated using simulation data extracted from the full three-dimensional volume. In contrast, data collected from the ocean are necessarily much more limited. In this section, we explore the sensitivity of the estimates of $\kappa$ when calculated with limited data. Note that we do not consider instrument error or biases introduced when converting measured quantities into physical quantities like the dissipation rate. Instead, we assume that the simulated field can be sampled perfectly at discrete points in space and focus on the influence of limited data availability.

The most common sampling strategy to infer $\kappa$ is to collect velocity, temperature, and/or conductivity along roughly vertical profiles. Measurements from distinct regions within one or more profiles are often averaged to reduce the uncertainty in the measurement. Here, we will calculate $\kappa$ using the methods described in the previous section based on a limited number of $1 \mathrm{D}$ vertical profiles extracted from the simulations. Note that the profiles that we use are taken instantaneously and are perfectly vertical. How well this describes oceanographic measurements depends on the fall speed of the instrument and the speed of the currents. Some platforms such as microstructure gliders make significantly inclined profiles, although these data are often analyzed in a similar way to free-falling profilers (e.g., Palmer et al. 2015).

We extract data from the simulations by randomly selecting a set of vertical profiles from a single threedimensional field. Since the simulations were sampled when the flow is in a statistically stationary state, sampling at different spatial locations should give the same statistical result as sampling at different time intervals. Treating a limited number of samples as independent vertical profiles is justified by the horizontal decorrelation of statistical quantities. For example, the horizontal autocorrelation length associated with the profile-averaged TKE dissipation rate $\langle\varepsilon\rangle_{z}$ drops to zero at a distance of $L_{z} / 2$ or $5 \mathrm{~m}$ in simulation $\mathrm{C}$ and a distance of $\sim 2 L_{z}$ or $\sim 10 \mathrm{~m}$ in simulations $\mathrm{A}$ and $\mathrm{B}$. Note that the properties of the large-scale flow in the simulations will be influenced by the forcing scheme used. In the ocean, where turbulence is associated with eddies, internal waves, and shear layers across a wide range of horizontal scales, the decorrelation distance between profile-averaged statistics could be much larger than $10 \mathrm{~m}$.

Before testing the methods for estimating $\kappa$ it is useful to quantify the variability in profile-averaged statistics induced by intermittent stratified turbulence. Figure 5 shows the probability density function (PDF) of the buoyancy flux, TKE, and potential energy dissipation rates, and squared Thorpe scale, each normalized by the corresponding volume average. Here the Thorpe scale is calculated by averaging the rms displacement over one vertical profile such that

$$
L_{T}^{z} \equiv\left\langle L_{d}^{2}\right\rangle_{z}^{1 / 2}
$$

Each PDF is calculated using the full 3D computational volume (i.e., vertical profiles were collected at every horizontal grid point). The Thorpe scale is squared for comparison with the other quantities since this quantity appears in the expression for $\kappa_{T}$.

The modes of the PDFs for all quantities shown in Fig. 5 are skewed toward values smaller than the volume average. It is well known in the turbulence literature that the point-wise TKE and variance dissipation rates are similarly skewed such that a small number of large values contribute significantly to the volume average (Pope 2000). The PDFs of local (pointwise) $\varepsilon$ and $\chi$ are typically assumed to be lognormal, following Kolmogorov (1962). De Bruyn Kops (2015) shows that distributions of local $\varepsilon$ and $\chi$ in stratified turbulence are well approximated by the lognormal model provided that $\mathrm{Re}_{b}>O(10)$. The TKE dissipation rate measured in the ocean thermocline is similarly skewed (Baker and Gibson 1987; Gregg et al. 1996). Evidently the intermittency inherent in the pointwise statistics extends to the profile-averaged statistics. 

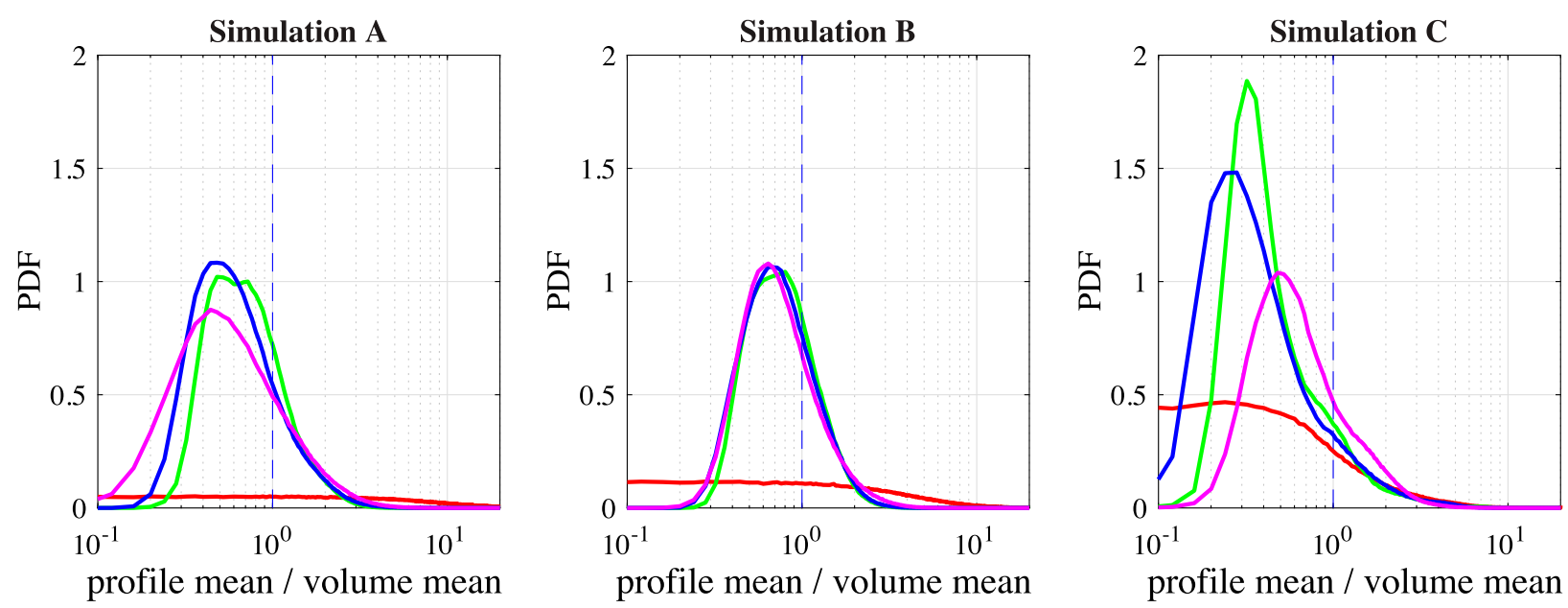

$$
-\langle B\rangle_{z} /\langle B\rangle_{V}-\langle\epsilon\rangle_{z} /\langle\epsilon\rangle_{V} \quad-\langle\chi\rangle_{z} /\langle\chi\rangle_{V} \quad-\left(L_{T}^{z} / L_{T}^{V}\right)^{2}
$$

FIG. 5. PDFs of the profile-averaged buoyancy flux $\langle B\rangle_{z}$, kinetic energy dissipation rate $\langle\varepsilon\rangle_{z}$, potential energy dissipation rate $\langle\chi\rangle_{z}$, and Thorpe scale $L_{T}^{z}$, each normalized by the volume average. There are a significant number of profiles with $\langle B\rangle_{z}<0$ that are not shown.

Here, we calculate $\varepsilon$ and $\chi$ using derivatives of all three velocity components and buoyancy in all three spatial directions. Field measurements of these quantities generally involve a subset of the velocity and/or gradient information and assumptions about the isotropy of the small-scale turbulence are invoked to fill in the missing information. The PDFs of the surrogates for $\varepsilon$ and $\chi$ based on a subset of the velocity and scalar gradients are significantly different from those of the exact quantities. In particular, the left side of the distributions of the surrogates tend toward exponential (Almalkie and de Bruyn Kops 2012a; de Bruyn Kops 2015) and the mean of the surrogates are significantly different from the exact values when $\mathrm{Re}_{b}$ is low (Hebert and de Bruyn Kops 2006b).

The variance associated with the buoyancy flux is much larger than the variance in other quantities. This appears to be associated with a large contribution from internal waves. Figure 2 shows regions with alternating sign of $w^{\prime} b^{\prime}$, indicating active exchange between kinetic and potential energy. The profile-averaged buoyancy flux is often negative (not shown in Fig. 5). As we will see below, the large variability in the profile-averaged buoyancy flux has significant implications for the estimates of $\kappa$.

Based on simulations of Kelvin-Helmholtz (KH) instability, Smyth et al. (2001) proposed that the ratio of the Ozmidov and Thorpe scales provides a measure of the "age" of a turbulent event. Their simulations started with a laminar stratified shear layer that was unstable to KH. During initial roll-up of the KH billows, they found that the Thorpe scale grows quickly, but dissipation remains low and as a result $L_{O} / L_{T} \ll 1$. In the later stages of their simulations, three-dimensional turbulence develops, and $L_{O} \sim L_{T}$. In simulations at substantially higher Reynolds number, Mashayek et al. (2017a) found analogous behavior, although they interpreted the (overall) monotonic increase in $L_{O} / L_{T}$ during the turbulent life cycle as being due to the relatively slower decrease in $L_{O}$ compared to $L_{T}$ during the turbulent decay phase of the life cycle. Mater et al. (2015) apply a similar argument to explain variations in $L_{O} / L_{T}$ in convectively generated turbulence. Observations reported in Smyth et al. (2001), Mater et al. (2015), and Mashayek et al. (2017a) all show relatively broad distributions of $L_{O} / L_{T}$.

Probability density functions of $L_{O}^{z} / L_{T}^{z}$ (where the dissipation rate and Thorpe displacement are calculated based on averages over individual vertical profiles) are shown in Fig. 6a. The peak in the distribution for simulation $\mathrm{C}$ is relatively close to the value of $L_{O} / L_{T}=0.8$ proposed by Dillon and Caldwell (1980), and the distribution qualitatively resembles the observations reported in Smyth et al. (2001). The ratio of the Ozmidov to Thorpe scale is somewhat smaller in simulations $\mathrm{A}$ and $\mathrm{B}$. This is consistent with the observations reported in Mater et al. (2015), which suggest that $L_{O} / L_{T}$ is not constant and depends on the properties of the flow. The spread in the distributions of $L_{O}^{z} / L_{T}^{z}$ suggests that the collection of profile-averaged statistics can be viewed as an ensemble of turbulent events as visualized in Fig. 1. 
(a)

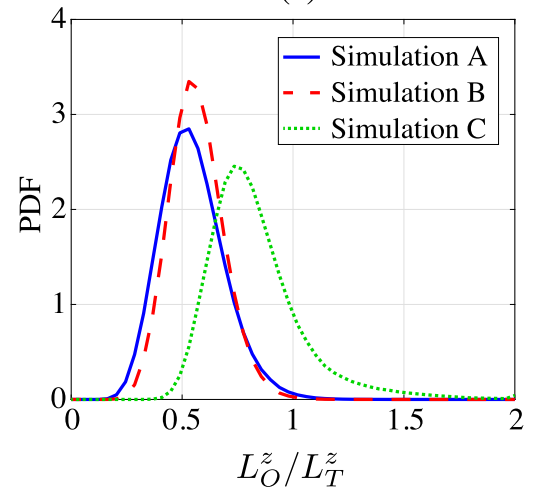

(b)

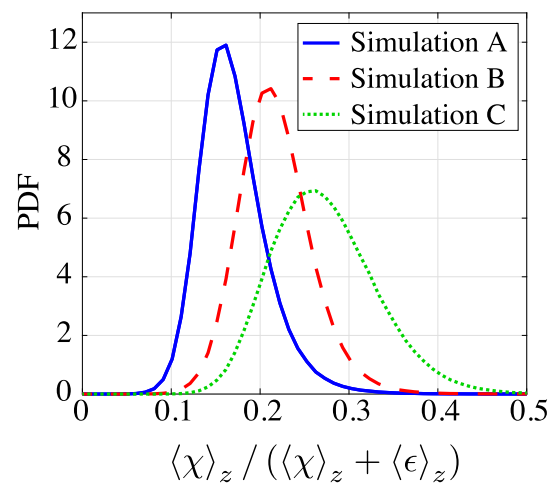

(c)

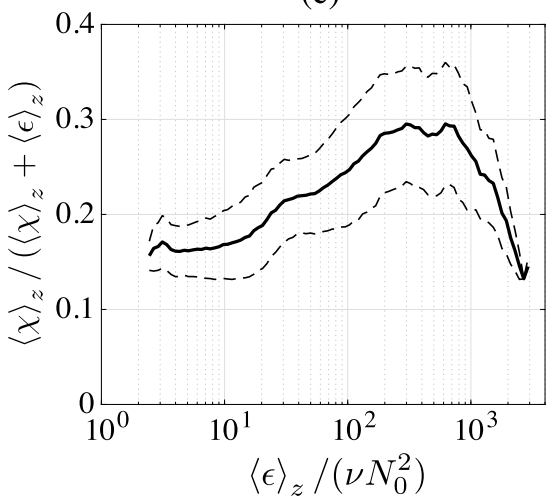

FIG. 6. Profile-averaged statistics: (a) PDF associated with the ratio of the Ozmidov and Thorpe scales, (b) PDF of mixing efficiency, and (c) mixing efficiency as a function of the buoyancy Reynolds number. Dashed lines in (c) indicate one standard deviation above and below the average value, and averaging bins with fewer than 10 profiles are not shown.

Figure 6b shows PDFs of mixing efficiency calculated using the profile-averaged dissipation rates, that is, $\langle\chi\rangle_{z} /\left(\langle\chi\rangle_{z}+\langle\varepsilon\rangle_{z}\right)$, which exhibits significant scatter about the volume average. The mean and mode of the distributions increase from simulation A to simulation C as the buoyancy Reynolds number increases. The mean values are somewhat larger than the canonical value of $1 / 6$, ranging from 0.18 in simulation A to 0.28 in simulation $\mathrm{C}$, although the spread about the mean is considerable. For example $\sim 22 \%$ of the profiles taken from simulation $\mathrm{C}$ have a mixing efficiency larger than 0.4 , although such large values do arise in idealized flows subject to strong $\mathrm{KH}$-like shear-driven overturning motions (see, e.g., Mashayek et al. 2013, 2017a).

Some recent studies have suggested that the mixing efficiency depends on the buoyancy Reynolds number, $\mathrm{Re}_{b} \equiv \varepsilon /\left(\nu N^{2}\right)$ (e.g., Shih et al. 2005; Mater and Venayagamoorthy 2014; Salehipour et al. 2016; Mashayek et al. 2017b; Monismith et al. 2018). Although there are differences in the details of various proposed scalings, most of the observations and simulations reported in these papers suggest a decrease in the mixing efficiency when $\mathrm{Re}_{b}$ exceeds a critical value. Figure $6 c$ shows the mixing efficiency plotted against $\mathrm{Re}_{b}$, with each quantity calculated from the profile-averaged dissipation rates. For small $\mathrm{Re}_{b}$ the mixing efficiency is very close to the value of 0.17 proposed by Osborn (1980), and consistent with previous numerical simulations (e.g., Shih et al. 2005). A peak in mixing efficiency for moderate values of $\mathrm{Re}_{b}$ as seen in Fig. $6 \mathrm{c}$ also occurs in some of the simulations from Shih et al. (2005; see also Mater and Venayagamoorthy 2014; Salehipour and Peltier 2015). Here, the mixing efficiency decreases with increasing buoyancy Reynolds number for $\mathrm{Re}_{b} \gtrsim 800$. This value is significantly larger than the threshold value found by Shih et al. (2005), but smaller than the value from observations reported in Lozovatsky and Fernando (2013) and well within the range of other simulations and observations (Mater and Venayagamoorthy 2014; Monismith et al. 2018).

Estimates of the vertical diffusivity calculated using sets of randomly selected vertical profiles are shown in Fig. 7. Specifically, when applied to $n$ vertical profiles, the vertical diffusivity estimated from the Osborn-Cox, Osborn, and Thorpe methods can be written

$$
\begin{aligned}
\kappa_{\mathrm{O}-\mathrm{C}}^{z, n} & =\frac{\langle\chi\rangle_{z, n}}{\left\langle N^{2}\right\rangle_{z, n}}, \quad \kappa_{O}^{z, n}=\Gamma \frac{\langle\varepsilon\rangle_{z, n}}{\left\langle N^{2}\right\rangle_{z, n}} \\
\kappa_{T}^{z, n} & =0.64 \Gamma\left(\left\langle L_{T}^{z}\right\rangle_{n}\right)^{2}\left\langle N^{2}\right\rangle_{z, n}^{1 / 2},
\end{aligned}
$$

respectively, where $\langle\cdot\rangle_{z, n}$ denotes an average over $n$ vertical profiles and $L_{T}^{z}$ is defined in Eq. (23). Similarly, the vertical diffusivity associated with the direct method applied to $n$ vertical profiles is

$$
\kappa_{d}^{z, n}=\frac{-\langle B\rangle_{z, n}}{\left\langle N^{2}\right\rangle_{z, n}} .
$$

Note that here $\left\langle N^{2}\right\rangle_{z, n}=N_{0}^{2}$ due to the periodicity of the computational domain. In Fig. 7 each estimate of $\kappa$ is dimensionalized such that the height of the vertical domain and the length of each profile is $5 \mathrm{~m}$. Solid colored lines show \pm 1 standard deviation about the mean, and the area between these curves is shaded to highlight the uncertainty associated with each estimate. Black dashed lines indicate the vertical diffusivity calculated with the volume-averaged buoyancy flux, that is, $\kappa_{d}^{V}$.

In all cases, $\boldsymbol{\kappa}_{d}^{z, n}$ converges very slowly to $\kappa_{d}^{V}$. Figure 8 shows the standard deviation of the averages of the buoyancy flux, kinetic and potential energy dissipation rates, and the squared Thorpe scale for a given number 


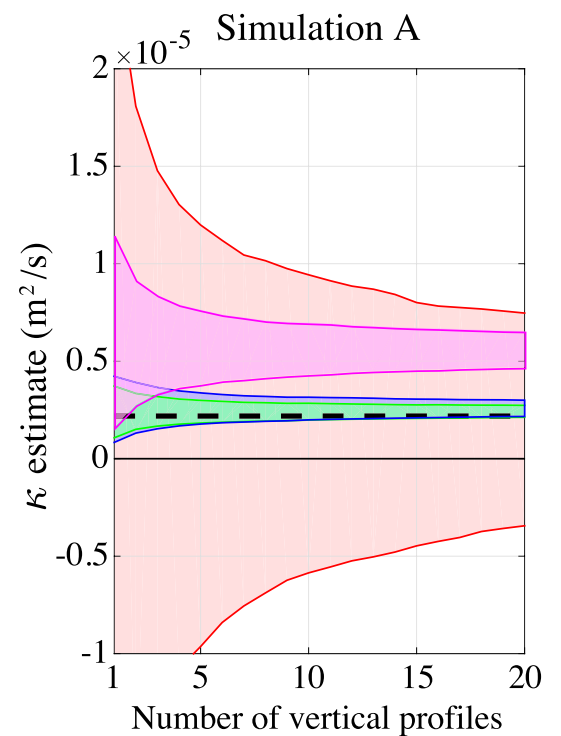

$\begin{array}{ccccc}5 & 25 & 50 & 75 & 100\end{array}$

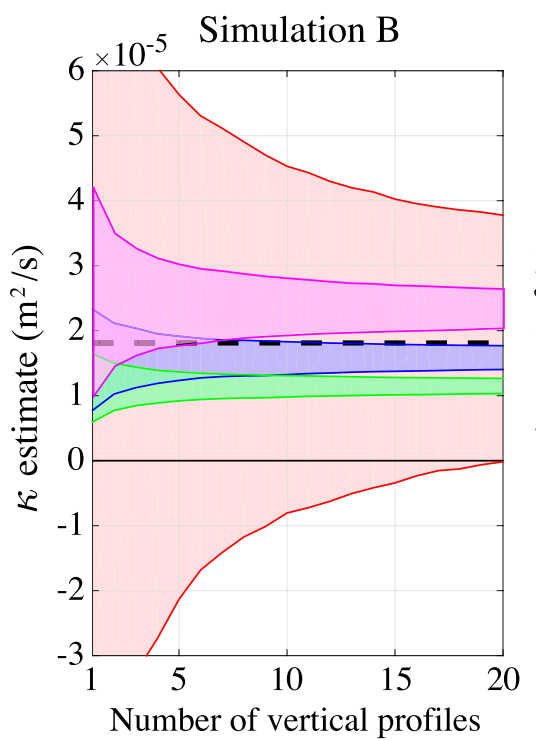

$\begin{array}{ccccc}5 & 25 & 50 & 75 & 100\end{array}$

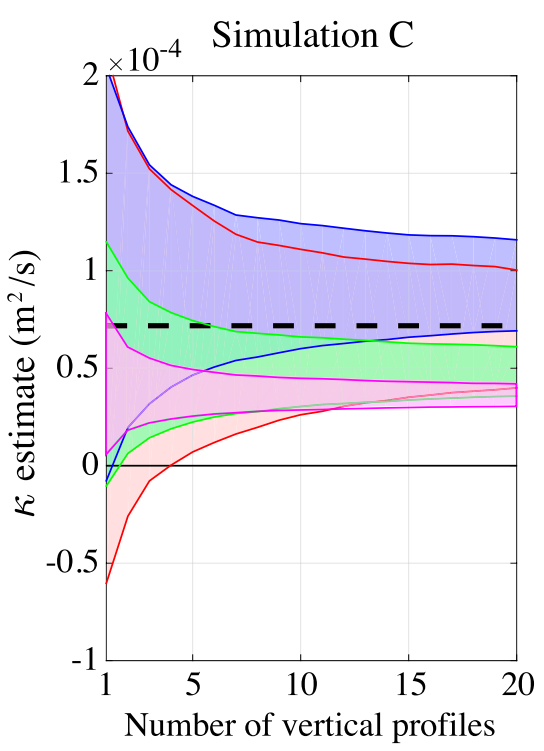

$\begin{array}{ccccc}5 & 25 & 50 & 75 & 100\end{array}$

$-\kappa_{O}^{z, n}-\kappa_{O-C}^{z, n}-\kappa_{T}^{z, n}-\kappa_{d}^{z, n}$

FIG. 7. Estimates of the vertical diffusivity using the Osborn (green), Osborn-Cox (blue), Thorpe (magenta), and direct methods (red), calculated using $n$ vertical profiles. Lines denote \pm 1 standard deviation about the mean, and the area between these limits is shaded. The dashed line indicates the vertical diffusivity calculated by directly averaging the flux over the full volume of the simulations as defined in Eq. (21). Note that the limits of the vertical axis are different in each panel.

of vertical profiles. In all cases the standard deviation decreases with the square root of the number of profiles (cf. with dashed line) as expected from the central limit theorem for independent random variables. However, even with 20 profiles, negative values of $\kappa_{d}^{z, n}$ are within one standard deviation of the mean in simulations $A$ and $\mathrm{B}$. The variance is smaller in simulation $\mathrm{C}$ where the flow is more turbulent.

The standard deviations associated with the profileaveraged dissipation rate and Thorpe scales are much smaller than the standard deviation of the buoyancy flux in simulations A and B. As seen in Fig. 4, the Osborn and Osborn-Cox methods give a relatively good estimate of $\kappa_{d}^{V}$ in these cases. Interestingly, the standard deviations of $\langle\varepsilon\rangle_{z, n}$ and $\langle\chi\rangle_{z, n}$ are significantly larger in simulation $\mathrm{C}$ and as a result the Osborn and Osborn-Cox methods require more profiles to converge in this case. Since simulation $\mathrm{C}$ is the most turbulent, having the largest dissipation rate, diffusivity, and buoyancy Reynolds number, the slow convergence of the Osborn and Osborn-Cox methods is unexpected and an explanation for this behavior is not immediately clear. In comparison, the Thorpe-scale method converges relatively quickly in simulation $\mathrm{C}$.

\section{e. Validity of assumptions underlying the Osborn and Osborn-Cox methods}

Remarkably, when applied to a limited number of vertical profiles, the Osborn and Osborn-Cox relations [Eqs. (17) and (13)] outperform their underlying assumptions. Figure 9 shows the normalized residual associated with the classic Osborn relation [Eq. (17), solid blue curve] and the classic Osborn-Cox relation [Eq. (13), dashed blue curve]. Here, the normalized residual is defined as the absolute value of the sum of the terms in each relation (with all terms on one side of the relevant equation) divided by the sum of the absolute values of each individual term.

For the Osborn model, we also evaluate the assumption that the turbulent flux coefficient $\Gamma$ is constant (solid green curve), and the assumed quasi-steady balance (unaffected by advection) in the TKE budget [Eq. (14), solid red curve]. The vertical and profile average is not shown in the legend for notational clarity but is applied to $\varepsilon, B, P$, and $N^{2}$ individually. We also evaluate the assumption underlying the Osborn-Cox model that the buoyancy variance budget reduces to a production/dissipation balance with $B \simeq \chi$ (dashed red curve). 

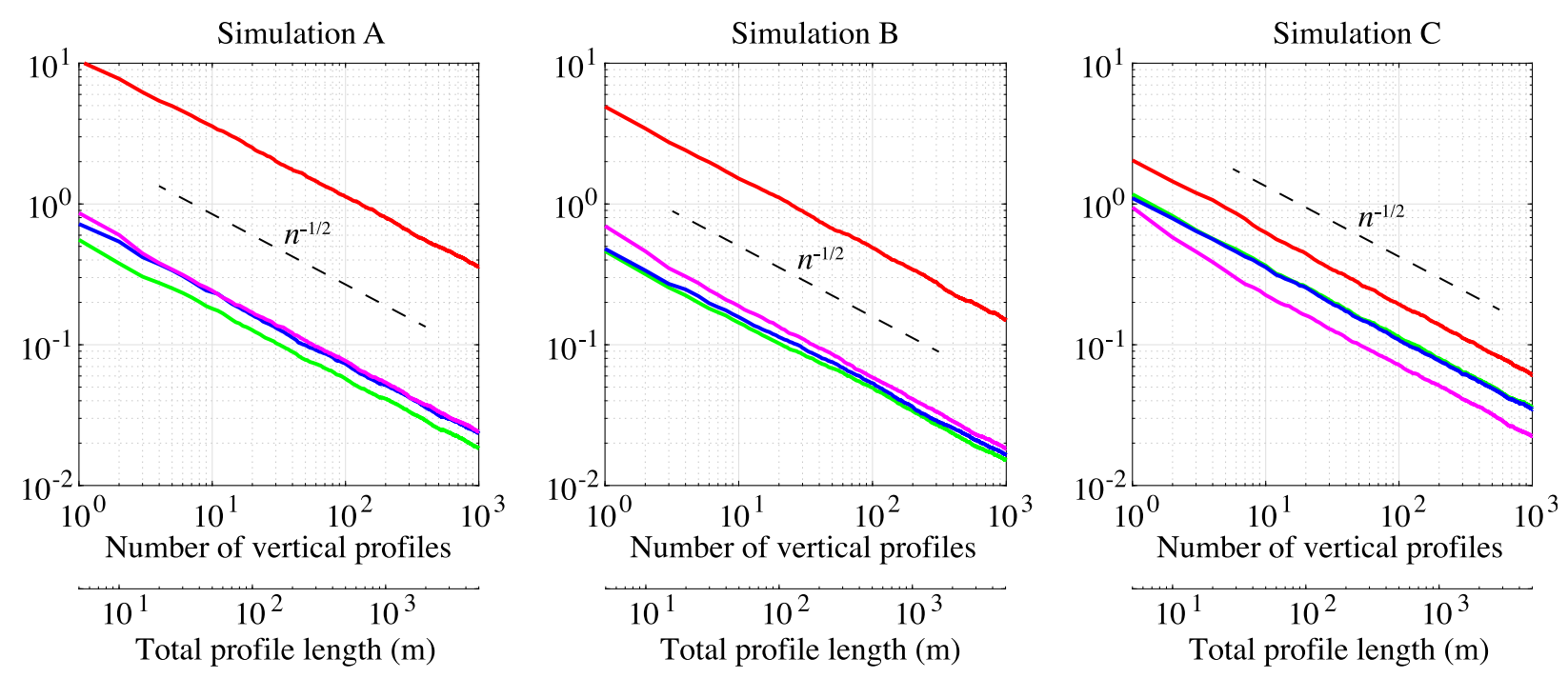

$-\operatorname{std}\left(\langle B\rangle_{z, n}\right) /\langle B\rangle_{V}-\operatorname{std}\left(\langle\epsilon\rangle_{z, n}\right) /\langle\epsilon\rangle_{V}-\operatorname{std}\left(\langle\chi\rangle_{z, n}\right) /\langle\chi\rangle_{V}-\operatorname{std}\left(\left\langle L_{T}^{z}\right\rangle_{n}^{2}\right) /\left(L_{T}^{V}\right)^{2}$

FIG. 8. Standard deviation associated with quantities averaged over $n$ vertical profiles, normalized by the 3D volume average. Dashed lines show the $n^{-1 / 2}$ scaling expected from the central limit theorem.

One might expect the error associated with the Osborn and Osborn-Cox relations to be at least as large as that of the worst assumption underlying these relations. Instead, the error associated with the Osborn relation is significantly less than the errors associated with the equations for the flux coefficient and TKE budgets for simulations $\mathrm{A}$ and $\mathrm{B}$. In case $\mathrm{C}$ the error in the Osborn relation is comparable to the error associated with the flux coefficient and smaller than the error associated with the TKE budget. A similar conclusion applies to the Osborn-Cox model where the Osborn-Cox relation (dashed blue curve in Fig. 9) significantly outperforms the assumption of production/dissipation balance in the buoyancy variance equation (dashed red curve) in simulations A and B.

An important difference between the Osborn and Osborn-Cox relations and the equations for the flux coefficient and the TKE and buoyancy variance budgets underlying these relations is that the buoyancy flux does not appear explicitly in the Osborn or Osborn-Cox relations. Figure 7 showed that the buoyancy flux exhibits very large scatter about its mean value, and this is particularly true in simulations A and B. One explanation for the relatively low normalized residuals associated with the Osborn and Osborn-Cox relations is that they are not by the reversible contributions of internal waves to the buoyancy flux. Indeed, central to the averaging at the heart of the Osborn method is the assumption that reversible processes in the buoyancy flux are filtered out, leaving only the irreversible component, capturing the actual mixing occurring within the flow.
Relatively recently, Salehipour and Peltier (2015) proposed a "generalized Osborn relation" using the framework introduced by Winters and D'Asaro (1996), designed explicitly to identify, as a function of time, the diapycnal diffusivity in terms of an appropriate definition for an inherently irreversible mixing efficiency. They showed that the diapycnal diffusivity $\kappa_{\rho}$ can be written as

$$
\kappa_{\rho}=\frac{\mathscr{E}}{1-\mathscr{E}} \frac{\varepsilon}{N_{*}^{2}}
$$

where $\mathscr{E}$ is the irreversible and instantaneous mixing efficiency defined in Caulfield and Peltier (2000) and $N_{*}$ is the buoyancy frequency calculated using the sorted density profile. Since this expression relies on quantities calculated from (volume) sorted data, it is a global measure of the mixing within the entire domain under consideration, but can in principle be calculated at every time instant within a temporally evolving flow. As the key parameters (such as an appropriately defined buoyancy Reynolds number and Richardson number) describing their simulated flow also vary in time, the results of their simulations, showing temporal variation of $\mathscr{E}$ can be interpreted as evidence that $\mathscr{E}$ depends on such parameters (Salehipour and Peltier 2015; Salehipour et al. 2016). Importantly, Eq. (26) does not rely on any assumptions aside from the Boussinesq approximation.

Salehipour and Peltier (2015) noted the clear structural similarity between Eq. (26) and the Osborn relation, Eq. (17). For strongly stratified flows with relatively small 

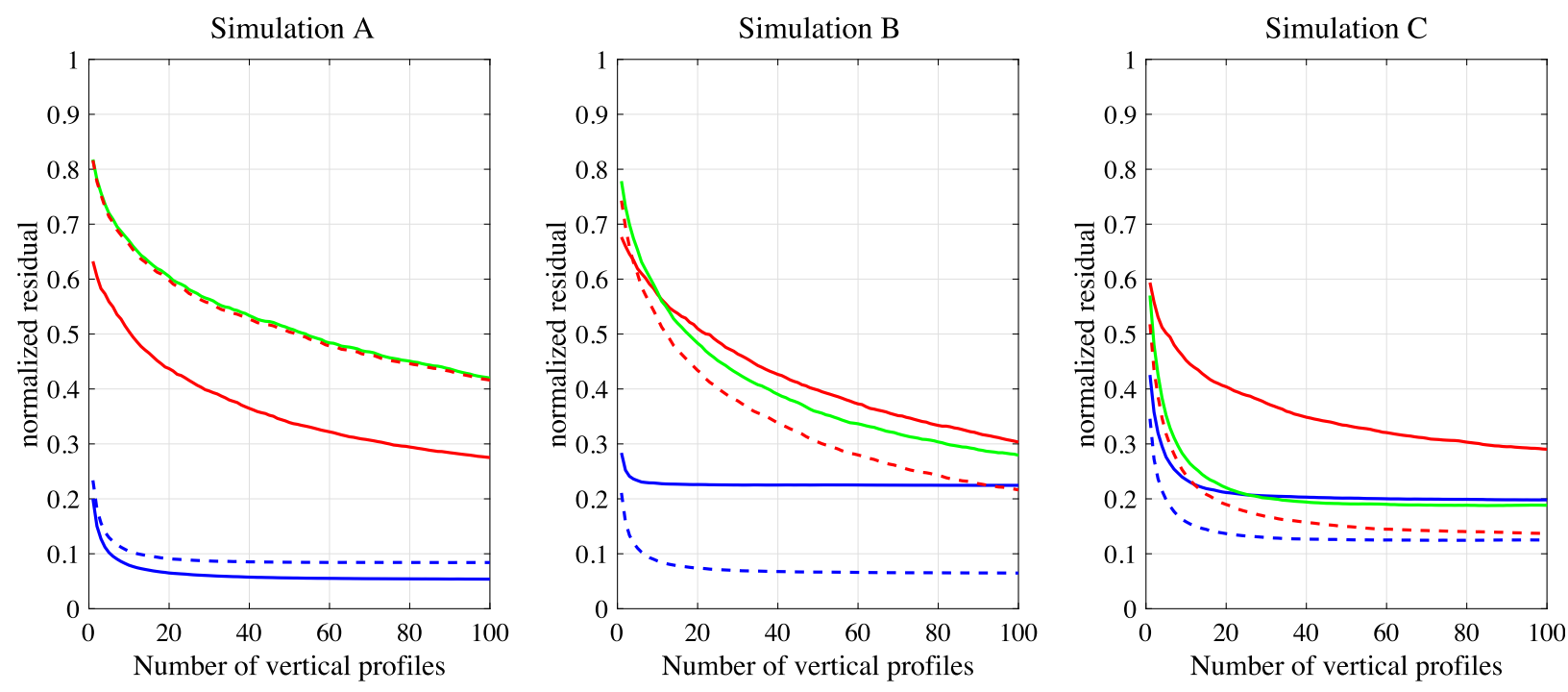

$$
\begin{array}{|cccc|}
\hline \text { Osborn model: }-\frac{\left|\frac{\Gamma \epsilon}{N^{2}}-\kappa_{d}^{V}\right|}{\left|\frac{\Gamma \epsilon}{N^{2}}\right|+\left|\kappa_{d}^{V}\right|} & -\frac{|\Gamma \epsilon+B|}{|\Gamma \epsilon|+|B|} & -\frac{|P-\epsilon+B|}{|P|+|\epsilon|+|B|} \\
\text { Osborn-Cox model: }--\frac{\left|\frac{\chi}{N^{2}}-\kappa_{d}^{V}\right|}{\left|\frac{\chi}{N^{2}}\right|+\left|\kappa_{d}^{V}\right|} & --\frac{|B+\chi|}{|B|+|\chi|}
\end{array}
$$

FIG. 9. Normalized residual associated with the Osborn and Osborn-Cox relations (blue) and several assumptions used to derive these relations (green and red). The values of $\varepsilon, \chi, B, P$, and $N^{2}$ correspond to an average across the vertical domain and for the specified number of vertical profiles, e.g., $\langle\varepsilon\rangle_{z, n}$, and the averaging operators are omitted for clarity.

isopycnal displacements one might anticipate that the globally sorted buoyancy frequency $N_{*} \simeq N$. To the extent that the flux coefficient $\Gamma$ in Eq. (17) approximates the irreversible flux coefficient $\mathscr{E} /(1-\mathscr{E})$, the Osborn relation could then provide a relatively robust approximation to the diapycnal diffusivity. Fundamentally, the key point is that assuming that the irreversible buoyancy flux is some fraction of the turbulent dissipation rate appears to be a reasonable assumption.

The dissipation rates of turbulent kinetic energy $\varepsilon$ and perturbation potential energy $\chi$ both represent irreversible losses from turbulence. As noted above, the partitioning of the total energy lost between these two terms is broadly consistent with the value of the flux coefficient used in the Osborn method, even though the theoretical arguments and assumptions presented by Osborn to justify this partitioning are not satisfied, not least due to the contaminating effects of reversible processes. The apparently robust partitioning between perturbation kinetic and potential energy dissipation might help explain why the Osborn method, applied using a limited number of vertical profiles, appears to be less prone to errors introduced by the presence of internal waves and other reversible processes than the failure of its underlying assumptions might suggest. It should be kept in mind that this discussion pertains to averaged quantities and that in local, transient mixing events the relative size of $\varepsilon$ and $\chi$ can vary substantially.

\section{Conclusions and discussion}

In this paper we tested the performance of the Osborn, Osborn-Cox, and Thorpe-scale methods using high resolution direct numerical simulations (DNS). The simulations used an idealized triply periodic computational domain with an imposed background stratification. Turbulence was forced using a deterministic body force added to the momentum equations. The simulations can be viewed as a model of turbulence in a small region embedded within the thermocline. Three simulations were run with varying stratification and turbulence levels, typical of conditions in the main and seasonal thermoclines.

When the Osborn and Osborn-Cox methods are applied to the volume-averaged TKE and perturbation potential energy dissipation rate, the resulting estimates of the vertical turbulent diffusivity $\left(\kappa_{O}^{V}\right.$ and $\left.\kappa_{\mathrm{O}-\mathrm{C}}^{V}\right)$ are within $40 \%$ of the value obtained directly from the volumeaveraged turbulent buoyancy flux $\kappa_{d}^{V}$. When the Thorpe scale is calculated using individual vertical profiles and then 
averaged over the full computational domain, the resulting estimate $\kappa_{T}^{V}$ is very close to $\kappa_{O}^{V}$ in simulation C but significantly overestimates $\kappa_{d}^{V}$ in simulations $\mathrm{A}$ and $\mathrm{B}$ with relatively small $\mathrm{Re}_{b}$. In simulation $\mathrm{A}, \kappa_{T}^{V}$ is more than 2.5 times larger than $\kappa_{d}^{V}$.

Consistent with previous simulations of forced stratified turbulence, we find that turbulence is inherently patchy and intermittent. For example, the PDFs of the dissipation rates of kinetic energy and buoyancy variance are skewed with a small number of very intense events, associated with vigorous, shear-driven overturnings. We find that this intermittency extends to the statistics averaged over one-dimensional vertical profiles, despite the fact that the simulations are set up such that each profile has the same average stratification.

This finding has important implications for the interpretation of limited observational datasets and for sampling strategies. For example, to ensure that the average dissipation rate can be correctly calculated, it would be necessary to ensure that enough of the extreme events are captured. The rates at which the various estimates of $\kappa$ converge to the values calculated with volume-averaged statistics depend on $\mathrm{Re}_{b}$. In general, the Osborn and Osborn-Cox methods converge relatively quickly in the simulations with small values of $\mathrm{Re}_{b}$, while the Thorpe-scale method converges somewhat faster in simulation $\mathrm{C}$ at larger $\mathrm{Re}_{b}$ than in simulations $\mathrm{A}$ and $\mathrm{B}$.

In comparison to the Osborn and Osborn-Cox methods, the diffusivity calculated directly from the vertical buoyancy flux using a small number of vertical profiles exhibits a very large scatter about the mean. Remarkably in simulations A and B, negative values of $\kappa$ are within one standard deviation of the average even when using 20 vertical profiles, each $5 \mathrm{~m}$ in length. The convergence to the mean is faster in simulation $\mathrm{C}$ where the flow is more turbulent. The slow convergence of the buoyancy flux for small $\mathrm{Re}_{b}$ appears to be due to large (and inherently reversible) contributions from internal waves. In an internal wave field the sign of $w^{\prime} b^{\prime}$ fluctuates as energy is transferred between the kinetic energy reservoir and the potential energy reservoir. A large averaging window (in space, in time or in ensemble) is required to eliminate these reversible contributions to the buoyancy flux.

Here, we have not tested the performance of finescale methods, which rely on measurements of internal waves. The large-scale forcing that was used to drive turbulence in the DNS was idealized and was not necessarily intended to replicate the properties of the finescale internal wave field. Simulations that simultaneously resolve a typical finescale internal wave spectrum (e.g., Gargett et al. 1981) while also resolving small-scale turbulence and mixing could be used to test (and perhaps improve) finescale methods.

Acknowledgments. The authors thank the Editor, Jim Moum, Matthew Alford, and an anonymous referee for constructive comments. We acknowledge the support of EPSRC under the Programme Grant EP/K034529/1 'Mathematical Underpinnings of Stratified Turbulence' (MUST), and from the European Research Council (ERC) under the European Union's Horizon 2020 research and innovation Grant 742480 'Stratified Turbulence And Mixing Processes' (STAMP), and also support from the U.S. Office of Naval Research under Grant N00014-15-1-2248. High performance computing resources were provided through the U.S. Department of Defense High Performance Computing Modernization Program by the Army Engineer Research and Development Center and the Army Research Laboratory under Frontier Project FP-CFD-FY14-007.

\section{REFERENCES}

Alford, M., and R. Pinkel, 2000a: Patterns of turbulent and double-diffusive phenomena: Observations from a rapidprofiling microconductivity probe. J. Phys. Oceanogr., 30, 833-854, https://doi.org/10.1175/1520-0485(2000)030<0833: POTADD $>2.0 . \mathrm{CO} ; 2$.

$\longrightarrow$, and — 2000b: Observations of overturning in the thermocline: The context of ocean mixing. J. Phys. Oceanogr., 30, 805-832, https://doi.org/10.1175/1520-0485(2000) 030<0805:OOOITT $>2.0$.CO;2.

Almalkie, S., and S. M. de Bruyn Kops, 2012a: Energy dissipation rate surrogates in incompressible Navier-Stokes turbulence. J. Fluid Mech., 697, 204-236, https://doi.org/ 10.1017/jfm.2012.53.

$\longrightarrow$, and $-2012 \mathrm{~b}$ : Kinetic energy dynamics in forced, homogeneous, and axisymmetric stably stratified turbulence. J. Turbul., 13, 1-29, https://doi.org/10.1080/14685248.2012.702909.

Baker, M. A., and C. H. Gibson, 1987: Sampling turbulence in the stratified ocean: Statistical consequences of strong intermittency. J. Phys. Oceanogr., 17, 1817-1836, https://doi.org/10.1175/ 1520-0485(1987)017<1817:STITSO>2.0.CO;2.

Caulfield, C. P., and W. R. Peltier, 2000: The anatomy of the mixing transition in homogeneous and stratified free shear layers. J. Fluid Mech., 413, 1-47, https://doi.org/10.1017/ S0022112000008284.

Comte-Bellot, G., and S. Corrsin, 1971: Simple Eulerian time correlation of full and narrow-band velocity signals in gridgenerated 'isotropic' turbulence. J. Fluid Mech., 48, 273-337, https://doi.org/10.1017/S0022112071001599.

de Bruyn Kops, S. M., 2015: Classical turbulence scaling and intermittency in stably stratified Boussinesq turbulence. J. Fluid Mech., 775, 436-463, https://doi.org/10.1017/jfm.2015.274.

_ and J. J. Riley, 2019: The effects of stable stratification on the decay of initially isotropic homogeneous turbulence. J. Fluid Mech., 860, 787-821, https://doi.org/10.1017/jfm.2018.888.

Dillon, T. M., and D. R. Caldwell, 1980: The Batchelor spectrum and dissipation in the upper ocean. J. Geophys. Res., 85, 1910-1916, https://doi.org/10.1029/JC085iC04p01910. 
Ferron, B., H. Mercier, K. Speer, A. Gargett, and K. Polzin, 1998: Mixing in the Romanche fracture zone. J. Phys. Oceanogr., 28, 1929-1945, https://doi.org/10.1175/1520-0485(1998)028<1929: MITRFZ $>2.0 . \mathrm{CO} ; 2$.

Gargett, A., and T. Garner, 2008: Determining Thorpe scales from ship-lowered CTD density profiles. J. Atmos. Oceanic Technol., 25, 1657-1670, https://doi.org/10.1175/2008JTECHO541.1.

—, P. Hendricks, T. Sanford, T. Osborn, and A. Williams, 1981: A composite spectrum of vertical shear in the upper ocean. J. Phys. Oceanogr., 11, 1258-1271, https://doi.org/10.1175/ 1520-0485(1981)011<1258:ACSOVS>2.0.CO;2.

Gibson, C. H., 1980: Fossil turbulence, salinity, and vorticity turbulence in the ocean. Marine Turbulence, J. C. Nihous, Ed., Elsevier Oceanography Series, Vol. 28, Elsevier, 221-257, https://doi.org/10.1016/S0422-9894(08)71223-6.

Gregg, M., 1989: Scaling turbulent dissipation in the thermocline. J. Geophys. Res., 94, 9686-9698, https://doi.org/10.1029/ JC094iC07p09686.

- 1999: Uncertainties and limitations in measuring $\varepsilon$ and $\chi_{t}$. J. Atmos. Oceanic Technol., 16, 1483-1490, https://doi.org/ 10.1175/1520-0426(1999)016<1483:UALIMA>2.0.CO;2.

—, D. Winkel, T. Sanford, and H. Peters, 1996: Turbulence produced by internal waves in the oceanic thermocline at mid and low latitudes. Dyn. Atmos. Oceans, 24, 1-14, https:// doi.org/10.1016/0377-0265(95)00406-8.

_- E. D'Asaro, J. Riley, and E. Kunze, 2018: Mixing efficiency in the ocean. Annu. Rev. Mar. Sci., 10, 443-473, https://doi.org/ 10.1146/annurev-marine-121916-063643.

Hebert, D. A., and S. M. de Bruyn Kops, 2006a: Predicting turbulence in flows with strong stable stratification. Phys. Fluids, 18, 066602, https://doi.org/10.1063/1.2204987.

- , and $-2006 \mathrm{~b}$ : Relationship between vertical shear rate and kinetic energy dissipation rate in stably stratified flows. Geophys. Res. Let., 33, L06602, https://doi.org/10.1029/ 2005 GL025071.

Henyey, F. S., J. Wright, and S. M. Flatté, 1986: Energy and action flow through the internal wave field: An eikonal approach. J. Geophys. Res., 91, 8487-8495, https://doi.org/ 10.1029/JC091iC07p08487.

Ivey, G. N., and J. Imberger, 1991: On the nature of turbulence in a stratified fluid. Part 1: The energetics of mixing. J. Phys. Oceanogr., 21, 650-658, https://doi.org/10.1175/1520-0485(1991) $021<0650$ :OTNOTI $>2.0 . \mathrm{CO} ; 2$.

Johnson, H. L., and C. Garrett, 2004: Effects of noise on Thorpe scales and run lengths. J. Phys. Oceanogr., 34, 2359-2372, https://doi.org/10.1175/JPO2641.1.

Kolmogorov, A. N., 1962: A refinement of previous hypotheses concerning the local structure of turbulence in a viscous incompressible fluid at high Reynolds number. J. Fluid Mech., 13, 82-85, https://doi.org/10.1017/S0022112062000518.

Large, W. G., J. C. McWilliams, and S. C. Doney, 1994: Oceanic vertical mixing: A review and a model with a nonlocal boundarylayer parameterization. Rev. Geophys., 32, 363-403, https://doi.org/ 10.1029/94RG01872.

Lindborg, E., 2006: The energy cascade in a strongly stratified fluid. J. Fluid Mech., 550, 207-242, https://doi.org/10.1017/ S0022112005008128.

Lozovatsky, I. D., and H. J. S. Fernando, 2013: Mixing efficiency in natural flows. Philos. Trans. Roy. Soc. London, 371, 20120213, https://doi.org/10.1098/RSTA.2012.0213.

MacKinnon, J., and M. Gregg, 2003: Mixing on the late-summer New England shelf - solibores, shear, and stratification. J. Phys. Oceanogr., 33, 1476-1492, https://doi.org/10.1175/1520-0485(2003) 033<1476:MOTLNE $>2.0$. CO;2.
Maffioli, A., and P. A. Davidson, 2016: Dynamics of stratified turbulence decaying from a high buoyancy Reynolds number. J. Fluid Mech., 786, 210-233, https://doi.org/10.1017/ jfm.2015.667.

Marra, J., R. Bidigare, and T. Dickey, 1990: Nutrients and mixing, chlorophyll and phytoplankton growth. Deep-Sea Res., 37A, 127-143, https://doi.org/10.1016/0198-0149(90)90032-Q.

Marshall, J., and K. Speer, 2012: Closure of the meridional overturning circulation through southern ocean upwelling. Nat. Geosci., 5, 171-180, https://doi.org/10.1038/ngeo1391.

Mashayek, A., C. P. Caulfield, and W. R. Peltier, 2013: Timedependent, non-monotonic mixing in stratified turbulent shear flows: Implications for oceanographic estimates of buoyancy flux. J. Fluid Mech., 736, 570-593, https://doi.org/10.1017/ jfm.2013.551.

,-- , and ——, 2017a: Role of overturns in optimal mixing in stratified mixing layers. J. Fluid Mech., 826, 522-552, https:// doi.org/10.1017/jfm.2017.374.

— , H. Salehipour, D. Bouffard, C. P. Caulfield, R. Ferrari, M. Nikurashin, W. R. Peltier, and W. D. Smyth, 2017b: Efficiency of turbulent mixing in the abyssal ocean circulation. Geophys. Res. Lett., 44, 6296-6306, https://doi.org/10.1002/ 2016 GL072452.

Mater, B. D., and S. K. Venayagamoorthy, 2014: A unifying framework for parameterizing stably stratified shear-flow turbulence. Phys. Fluids, 26, 036601, https://doi.org/10.1063/1.4868142.

—_ —_, L. St. Laurent, and J. N. Moum, 2015: Biases in Thorpe-scale estimates of turbulence dissipation. Part I: Assessments from large-scale overturns in oceanographic data. J. Phys. Oceanogr., 45, 2497-2521, https://doi.org/ 10.1175/JPO-D-14-0128.1.

Monismith, S. G., J. R. Koseff, and B. L. White, 2018: Mixing efficiency in the presence of stratification: When is it constant? Geophys. Res. Lett., 45, 5627-5634, https://doi.org/10.1029/ 2018 GL077229.

Moum, J. N., 1996: Energy-containing scales of turbulence in the ocean thermocline. J. Geophys. Res., 101, 14095-14109, https://doi.org/10.1029/96JC00507.

—, M. Gregg, R. Lien, and M. Carr, 1995: Comparison of turbulence kinetic energy dissipation rate estimates from two ocean microstructure profilers. J. Atmos. Oceanic Technol., 12, 346-366, https://doi.org/10.1175/1520-0426(1995)012<0346: COTKED $>2.0 . \mathrm{CO} ; 2$.

Osborn, T. R., 1980: Estimates of the local-rate of vertical diffusion from dissipation measurements. J. Phys. Oceanogr., 10, 83-89, https://doi.org/10.1175/1520-0485(1980) $010<0083$ :EOTLRO $>2.0 . \mathrm{CO} ; 2$.

—_, and C. S. Cox, 1972: Oceanic fine structure. Geophys. Astrophys. Fluid Dyn., 3, 321-345, https://doi.org/10.1080/ 03091927208236085.

Overholt, M. R., and S. B. Pope, 1998: A deterministic forcing scheme for direct numerical simulations of turbulence. Comput. Fluids, 27, 11-28, https://doi.org/10.1016/S0045-7930(97) 00019-4.

Pacanowski, R., and S. Philander, 1981: Parameterization of vertical mixing in numerical models of tropical oceans. J. Phys. Oceanogr., 11, 1443-1451, https://doi.org/10.1175/1520-0485(1981) 011<1443:POVMIN>2.0.CO;2.

Palmer, M., G. Stephenson, M. Inall, C. Balfour, A. Düsterhus, and J. Green, 2015: Turbulence and mixing by internal waves in the Celtic Sea determined from ocean glider microstructure measurements. J. Mar. Syst., 144, 57-69, https://doi.org/10.1016/ j.jmarsys.2014.11.005. 
Polzin, K. L., J. M. Toole, and R. W. Schmitt, 1995: Finescale parameterizations of turbulent dissipation. J. Phys. Oceanogr., 25, 306-328, https://doi.org/10.1175/1520-0485(1995) $025<0306$ :FPOTD $>2.0$. CO; 2 .

Pope, S. B., 2000: Turbulent Flows. Cambridge University Press, $806 \mathrm{pp}$.

Portwood, G. D., S. M. de Bruyn Kops, J. R. Taylor, H. Salehipour, and C. P. Caulfield, 2016: Robust identification of dynamically distinct regions in stratified turbulence. J. Fluid Mech., 807, R2, https://doi.org/10.1017/jfm.2016.617.

Rao, K. J., and S. M. de Bruyn Kops, 2011: A mathematical framework for forcing turbulence applied to horizontally homogeneous stratified flow. Phys. Fluids, 23, 065110, https:// doi.org/10.1063/1.3599704.

Riley, J. J., and S. M. de Bruyn Kops, 2003: Dynamics of turbulence strongly influenced by buoyancy. Phys. Fluids, 15, 2047-2059, https://doi.org/10.1063/1.1578077.

Rorai, C., P. D. Mininni, and A. Pouquet, 2014: Turbulence comes in bursts in stably stratified flows. Phys. Rev., 89E, 043002, https://doi.org/10.1103/PHYSREVE.89.043002.

Salehipour, H., and W. Peltier, 2015: Diapycnal diffusivity, turbulent Prandtl number and mixing efficiency in Boussinesq stratified turbulence. J. Fluid Mech., 775, 464-500, https://doi.org/ 10.1017/jfm.2015.305.

- - W. R. Peltier, C. B. Whalen, and J. A. MacKinnon, 2016: A new characterization of the turbulent diapycnal diffusivities of mass and momentum in the ocean. Geophys. Res. Lett., 43, 3370-3379, https://doi.org/10.1002/ 2016GL068184.

Scotti, A., 2015: Biases in Thorpe-scale estimates of turbulence dissipation. Part II: energetics arguments and turbulence simulations. J. Phys. Oceanogr., 45, 2522-2543, https://doi.org/ 10.1175/JPO-D-14-0092.1.

Shih, L. H., J. R. Koseff, G. N. Ivey, and J. H. Ferziger, 2005: Parameterization of turbulent fluxes and scales using homogeneous sheared stably stratified turbulence simulations. J. Fluid Mech., 525, 193-214, https://doi.org/10.1017/ S0022112004002587.
Smyth, W. D., and J. N. Moum, 2000: Length scales of turbulence in stably stratified mixing layers. Phys. Fluids, 12, 1327-1342, https://doi.org/10.1063/1.870385.

,-- , and D. R. Caldwell, 2001: The efficiency of mixing in turbulent patches: Inferences from direct simulations and microstructure observations. J. Phys. Oceanogr., 31, 1969-1992, https://doi.org/10.1175/1520-0485(2001)031<1969:TEOMIT> 2.0.CO;2.

Sreenivasan, K. R., 1998: An update on the energy dissipation rate in isotropic turbulence. Phys. Fluids, 10, 528-529, https:// doi.org/10.1063/1.869575.

— , and R. A. Antonia, 1997: The phenomenology of small-scale turbulence. Annu. Rev. Fluid Mech., 29, 435-472, https://doi.org/ 10.1146/annurev.fluid.29.1.435.

Thorpe, S. A., 1977: Turbulence and mixing in a Scottish loch. Philos. Trans. Roy. Soc. London, 286A, 125-181, https://doi.org/ 10.1098/rsta.1977.0112.

_ 2005: The Turbulent Ocean. Cambridge University Press, $439 \mathrm{pp}$.

Waite, M. L., 2011: Stratified turbulence at the buoyancy scale. Phys. Fluids, 23, 066602, https://doi.org/10.1063/1.3599699.

Wang, L. P., S. Y. Chen, J. G. Brasseur, and J. C. Wyngaard, 1996: Examination of hypotheses in the Kolmogorov refined turbulence theory through high-resolution simulations. Part 1 . Velocity field. J. Fluid Mech., 309, 113-156, https://doi.org/ 10.1017/S0022112096001589.

Warhaft, Z., 2000: Passive scalar in turbulent flows. Annu. Rev. Fluid Mech., 32, 203-240, https://doi.org/10.1146/annurev.fluid.32.1.203.

Waterhouse, A. F., and Coauthors, 2014: Global patterns of diapycnal mixing from measurements of the turbulent dissipation rate. J. Phys. Oceanogr., 44, 1854-1872, https://doi.org/10.1175/ JPO-D-13-0104.1.

Winters, K. B., and E. A. D'Asaro, 1996: Diascalar flux and the rate of fluid mixing. J. Fluid Mech., 317, 179-193, https://doi.org/ 10.1017/S0022112096000717.

Wunsch, C., and R. Ferrari, 2004: Vertical mixing, energy, and the general circulation of the oceans. Annu. Rev. Fluid Mech., 36, 281-314, https://doi.org/10.1146/annurev.fluid.36.050802.122121. 